\title{
Increased expression of PSME2 is associated with clear cell renal cell carcinoma invasion by regulating BNIP3-mediated autophagy
}

\author{
XIAOYUN WANG ${ }^{1,2^{*}}$, FENGBO WU $^{1 *}$, YUTONG DENG $^{3}$, JINLONG CHAI $^{1}$, \\ YUEHUA ZHANG ${ }^{1}, \mathrm{GU} \mathrm{HE}^{1}$ and XIANG $\mathrm{LI}^{2}$ \\ ${ }^{1}$ State Key Laboratory of Biotherapy and Department of Pharmacy, ${ }^{2}$ Department of Urology, West China Hospital, \\ Sichuan University and Collaborative Innovation Center of Biotherapy, Chengdu, Sichuan 610041; \\ ${ }^{3}$ College of Environmental Sciences and Engineering, Peking University, Beijing 100871, P.R. China
}

Received May 17, 2021; Accepted October 11, 2021

DOI: $10.3892 / \mathrm{ijo} .2021 .5286$

\begin{abstract}
Previous studies have showed that proteasome activator complex subunit 2 (PSME2) may play a role in some types of cancer. However, the involvement of PSME2 in clear cell renal cell carcinoma (ccRCC) remains unknown. The aim of the present study was to assess the poorly understood function of PSME2 expression in renal carcinoma. Using bioinformatics analysis, PSME2 mRNA expression profiles were investigated, along with its potential prognostic value and its functional enrichment. Signaling pathways and putative hub genes associated with PSME2 in ccRCC were identified. Based on the bioinformatics analysis results, immunohistochemistry of human ccRCC samples and renal carcinoma cell lines (CAKI-1 and 786-O) transfected with short interfering RNA targeting PSME2 were analyzed using western blot analysis, reverse transcription-quantitative PCR, immunofluorescence, and Cell Counting Kit-8, Transwell and transmission electron microscope assays. The results showed that when PSME2 expression was knocked down, the invasive abilities of the tumor cell lines were reduced, while autophagy was enhanced. The present study demonstrated that PSME2 was associated with the invasion ability of ccRCC cell lines by inhibiting BNIP3-mediated autophagy. In summary, PSME2 could be used as a prognostic factor and a promising therapeutic target in ccRCC.
\end{abstract}

Correspondence to: Professor Gu He, State Key Laboratory of Biotherapy and Department of Pharmacy, West China Hospital, Sichuan University and Collaborative Innovation Center of Biotherapy, 37 GuoXueXiang, Chengdu, Sichuan 610041, P.R. China E-mail: hegu@scu.edu.cn

Professor Xiang Li, Department of Urology, West China Hospital, Sichuan University and Collaborative Innovation Center of Biotherapy, 37 GuoXueXiang, Chengdu, Sichuan 610041, P.R. China E-mail: xiangli.87@163.com

*Contributed equally

Key words: proteasome activator complex subunit 2, BCL2 interacting protein, clear cell renal cell carcinoma, invasion, autophagy

\section{Introduction}

The proteasome, also known as a core particle (CP) or $20 \mathrm{~S}$ proteasome, is a large, major intracellular, multi-catalytic protease in eukaryotic cells (1). The proteasome is responsible for the degradation of most cellular proteins via non-lysosomal proteolytic pathways (2-4). There are four different proteasome regulatory complexes (PA700, PA28, PA28 $\gamma$ and PA200), which affect proteasomal activity (5). PA700, also termed the regulatory complex or $19 \mathrm{~S}$, couples with the $20 \mathrm{~S}$ proteasome to form the $26 \mathrm{~S}$ proteasome (5). The $26 \mathrm{~S}$ proteasome is responsible for breaking down ubiquitinated protein substrates in an ATP-dependent manner (6). PA28, an 11S protein regulator of the 20S proteasome, comprises of two distinct but homologous polypeptides, termed PA28 $\alpha$ and PA28 $\beta$. PA28 $\alpha$ and PA28 $\beta$ are encoded by small diverse genes, proteasome activator complex subunit (PSME)1 and PSME2, respectively (7-9). PA28 $\alpha \beta$ activates the proteasome by binding to the cylinder end of the 20S proteinase and opening the catalytic center (10-12). PA28 $\gamma$, encoded by PSME3, forms a homoheptamer and binds to the $\alpha$-ring of the CP (13). PA200 is encoded by PSME4 and, similarly to PA28, binds to the end of the 20S CP $(5,14,15)$.

PA28 $\alpha \beta$ is expressed primarily in the cytoplasm, whereas PA28 $\gamma$ and PA200 are highly abundant within the nucleus $(16,17)$. PA2 $\alpha \alpha$ and $\beta$ subunits are highly inducible by IFN $-\gamma$, and are abundant in the liver, lung and spleen, but has limited expression in the brain. By contrast, PA28 $\gamma$ is not induced by IFN- $\gamma$, and is abundant in the brain and has moderate expression in other organs, such as the spleen $(5,18)$. PA200 exists in all mammalian tissues, and is expressed abundantly in the testis and sperm (19). PA28 modulates the proteasome-catalyzed products of the major histocompatibility class I antigenic peptides to present to cytotoxic $\mathrm{T}$ lymphocytes, inferring its association with the immune response (20-22). Previous studies have found that PA28 could activate the hydrolysis of small non-ubiquitinated peptides and has protective functions against oxidative stress (23-25). A previous study demonstrated that PA28 was associated with colon cancer, while other studies reported that PA28 $\beta$ plays a role in some cancers $(26,27)$. For example, using two-dimensional polyacrylamide gel electrophoresis-based proteomics, Ebert et al (28) showed that human PA28 $\beta$ protein expression was increased in gastric cancer. Perroud et al (29) 
also discovered that the protein expression levels of PA28 $\beta$ was upregulated in clear cell renal cell carcinoma (ccRCC) compared with that in normal kidney tissue, as determined using proteomics. The mRNA expression levels of PA28 $\beta$ were markedly elevated in cutaneous skin melanoma and Burkitt lymphoma $(14,20)$. Higher protein expression levels of PA28 $\beta$ were detected in primary breast tumors compared with that in lymph node metastasis $(30)$. However, Huang et al $(31,32)$ and Zheng et al (33) showed that the protein expression level of PA28 $\beta$ was decreased in gastric adenocarcinoma (GA) and this decreased expression was associated with poorer GA differentiation. Kim et al (34) confirmed that PA28 $\beta$ expression was decreased in lung cancer. In human esophageal squamous cell carcinoma, PA28 $\beta$ expression was significantly lower (35). In conclusion, PA28 $\beta$ expression is differently associated with several types of cancer.

The present study aimed to investigate PSME2, as a biomarker for ccRCC using the protein expression patterns of kidney renal clear cell carcinoma (KIRC) tissues and matched adjacent normal tissue. In addition, the association between PSME2 expression level and BCL2 interacting protein (BNIP) 3-mediated autophagy in ccRCC was also analyzed. The findings suggest that PSME2 may be a candidate target for kidney cancer therapy.

\section{Materials and methods}

Samples and data processing. The mRNA expression data of tumor and normal adjacent tissue from patients with KIRC were downloaded from two platforms, including KIRC cohort from TCGA (https://www.cancer.gov/about-nci/organization/ccg/research/structural-genomics/tcga). TCGA database comprises of a large number of gene expression data and is a useful resource for understanding the molecular basis of cancer. In the present study, a dataset containing 533 tumor samples and 72 paired normal samples was downloaded. A paired t-test was used to compare the expression level of four PSME genes between the tumor tissues and adjacent normal tissues. The Oncomine database, which is a publicly accessible online cancer microarray database (https://www.oncomine.org/resource/login.html) (36), was used to analyze the mRNA expression level of PSME2 in tumor $(n=486)$ and normal $(n=338)$ tissues from different types of cancer. During the analysis, the numbers in the boxes indicate the number of GEO datasets containing differential expressed PSME2; the red color indicates the expression of PSME2 ranked in top $10 \%$ of all genes and blue color indicates PSME2 ranked in bottom $10 \%$ of all genes. Various threshold parameters were set as follows: $\mathrm{P}<0.01$; fold change $>2$; gene ranking, all and data type, mRNA. The RNA Sequencing dataset, from the two datasets were expressed as fragments per kilobase per million mapped reads and were $\log _{2}$ transformed after the addition of one $\left[\log _{2}(\mathrm{x}+1)\right]$ prior to analysis.

Survival analysis. Kaplan-Meier plotter was used to calculate the survival time in patients with KIRC in each dataset. The survival probability, including overall survival (OS), disease-specific survival (DSS), and progression-free survival (PFS) times, were evaluated for patients with high or low mRNA expression levels of PSME2, based on the best group separation. The log-rank test was used and $\mathrm{P}<0.05$ was considered to indicate a statistically significant difference. Survival analyses were performed using R software v3.6.3 (https://www.r-project.org/).

Bioinformatics analysis. The top $25 \%$ of genes with the largest variance were used to perform a weighted gene co-expression network analysis (WGCNA) with the 'WGCNA' package in $\mathrm{R}$ software (37). The correlation coefficient and P-value of the module characteristic genes with tumor and normal tissues were calculated using Pearson correlation coefficient (PCC) algorithm. The absolute value of the correlation coefficient was $\geq 0.5$, and $\mathrm{P}<0.05$ was used to screen the modules associated with each trait. Gene dendrogram construction and module identification was performed with the dynamic sheer method (38). Further study of the brown module with the tumor tissue, which was significantly associated with PSME2 expression level, was performed for functional enrichment analyses.

Gene Ontology (GO) and Kyoto Encyclopedia of Genes and Genomes (KEGG) analyses were used to identify the biological functions in the co-expression module. The Database for Annotation, Visualization and Integrated Discovery (DAVID; version 6.8) $(39,40)$ database is a comprehensive set of functional annotation tools for high-throughput gene function enrichment analysis. The biological processes and enriched pathways of the proteins encoded by the candidate genes were analyzed using DAVID. $\mathrm{P}<0.05$ was set as the cut-off criterion.

Gene Set Enrichment Analysis (GSEA; https://www. gsea-msigdb.org/gsea/index.jsp) was performed to screen the GO terms and KEGG pathways that may be associated with PSME2 based on the 6,290 curated gene datasets in the database. In this analysis, all of the ccRCC and normal samples were analyzed and a total of 1,097 kidney cancer samples were divided into two groups based on the expression level of PSME2; low expression level was defined as $\log _{2}(\mathrm{TPM}+1)<6.2$, while high expression level was defined as $\left.\log _{2}(\mathrm{TPM}+1)>6.2\right)$. The cut-off values for GSEA were false discovery rate $(\mathrm{FDR})<0.25$ and $\mathrm{P}<0.05$.

Bioinformatics analyses were performed using the $\mathrm{R}$ programming language (https://www.r-project.org). Cox proportional hazard regression analysis was used to evaluate the association between the risk score and OS, DSS and PFS times. Receiver operating characteristic (ROC) analysis was used to determine the specificity and sensitivity of PSME mRNA expression level in the KIRC tissues. The area under the curve (AUC) was used as an indicator of reliability.

Tissue microarray (TMA) and IHC. The human ccRCC TMA (cat. no. HKidE150CS01) was purchased from Shanghai Xinchao Biological Technology Co., Ltd. The TMA contained 150 tumors and matched adjacent normal kidney tissues $(0.5-2.0 \mathrm{~cm})(41)$ from 75 patients, including clear cell carcinoma, chromophobe cell carcinoma, papillary carcinoma and urothelial carcinoma. The clinical variables, including year of diagnosis, age at diagnosis, survival status and sex were collected, in an unbiased manner. These tissues were taken during renal cell carcinoma surgical resection between July 2006 and February 2007, and the last follow-up day was in August 2015. Kidney function and tumor stages were assessed according to the 2010 International Union Against Cancer Tumor-Node-Metastasis (TNM) classification system (42), and curative resection was defined as previously described (43). The following inclusion criteria were used: Only one tumor lesion, 
absence of any metastasis and aged 18-70 years. The following exclusion criteria were used: Liver or kidney function insufficient [includes one of the following: Alanine aminotransferase (ALT) $<7.0$ or $>40.0 \mathrm{U} / 1$; aspartate aminotransferase (AST) $<13.0$ or $>35.0 \mathrm{U} / \mathrm{l}$; AST/ALT $>1$; alkaline phosphatase (ALP) $<40.0$ or $>110.0 \mathrm{U} / 1$; serum creatinine $>133 \mu \mathrm{mol} / \mathrm{l}$; urine $\mathrm{pH}<5.0$ or $>8.0$; urine protein $>1.5 \mathrm{~g} / 24 \mathrm{~h}$; or positive urine occult blood test), receiving chemotherapy/radiotherapy or difficult to follow up. Clinicopathological data of the patients with RCC were collected from medical records at The West China Hospital (Sichuan, China). Survival information was obtained from the Social Security Death Index, telephone interviews and medical records, and all pathological data were originally assessed by two pathologists. The present study was performed in accordance with medical ethics and was approved by the West China Hospital of Sichuan University Biomedical Research Ethics Committee (Sichuan, China). Written informed consent was obtained from all participants before collection of the specimens. The IHC staining was performed according to the manufacturer's protocol using the primary antibody against PSME2 (cat. no. ab183727; 1:150 dilution; Abcam), as previously described (44). The omission of primary antibodies was used as the negative control, and a brown color was considered as positive staining.

Cell culture and transfection. The HK-2 cell line was purchased from the China Center for Type Culture Collection from Wuhan University (Hubei, China) and was cultured in DMEM/F12 supplemented with 10\% FBS (both from Gibco; Thermo Fisher Scientific, Inc.). The 786-O, CAKI-1, ACHN and A498 cell lines were purchased from the State Key Laboratory of Biotherapy of Sichuan University (Sichuan, China). The 786-O cell line was cultured with RPMI-1640 medium, containing 10\% FBS, while the CAKI-1 cell line was cultured in McCoy's 5a (Modified) medium supplemented with $10 \%$ FBS and the ACHN and A498 cell lines were cultured in EMEM, containing 10\% FBS (all from Gibco; Thermo Fisher Scientific, Inc.). All medium contained $1 \%$ penicillin/streptomycin solution (Hyclone; Cytiva). All the cell lines were maintained at $37^{\circ} \mathrm{C}$ in a humidified incubator with $5 \% \mathrm{CO}_{2}$.

The small interfering (si)RNA negative control (scrambled NC) (sense, 5'-UUCUCCGAACGUGUCACGUTT-3' and antisense, 5'-ACGUGACACGUUCGGAGAATT-3'), siPSME2\#1 (sense, 5'-CAAGGAUGAUGAGAUGGAAAC-3' and antisense, 5'-UUCCAUCUCAUCAUCCUUGGG-3'), siPSME2\#2 (sense, 5'-CAGAGAUCUAGCGACUGAAGC-3' and antisense 5'-UUCAGUCGCUAGAUCUCUGGU-3'), siPSME2\#3 (sense, 5'-CCAAGAUUGAAGAUGGAAAUG-3' and antisense, 5'-UUUCCAUCUUCAAUCUUGGGG-3') and siBNIP3 (sense, 5'-UACUGCUGGACGCACAGCAdTdT-3' and antisense, 5'-UGCAGUGCGUCCAGCAGUAdTdT-3') were purchased from Shanghai GenePharma Co., Ltd. For RNA interference, the CAKI-1 and 786-O cell lines were transfected with siNC or siPSME2 and/or siBNIP3 (50 nM) using Lipofectamine ${ }^{\circledR} 3000$ (Invitrogen; Thermo Fisher Scientific, Inc.) according to manufacturer's recommendations. After transfection for 24 and $48 \mathrm{~h}$, the cells were used for subsequent experimentation.

To overexpress BNIP3 in the CAKI-1 cell lines, both cell lines were transfected with expressing vector or the empty control vector pLKO.1-puro (50 nM; Sigma-Aldrich; Merck KGaA) using Lipofectamine ${ }^{\circledR} 3000$ (Invitrogen; Thermo Fisher Scientific, Inc.). After transfection at $37^{\circ} \mathrm{C}$ in $5 \%$ a humidified incubator with $\mathrm{CO}_{2}$ for 12,24 and $48 \mathrm{~h}$, the cells were selected with $5 \mu \mathrm{g} / \mathrm{ml}$ puromycin (Sigma-Aldrich; Merck KGaA) after 3-5 days. Puromycin-resistant clones were tested for their ability to overexpress BNIP3 and used for subsequent experimentation.

Transwell invasion assay. The cell invasion assays were performed using a Transwell chamber (24-well; $8 \mu \mathrm{m}$ pore size; Corning, Inc.). Matrigel matrix on ice (Corning, Inc.) was diluted 1:8 with pre-cooled medium and coated on the upper chamber surface of the Transwell membrane for $0.5-1 \mathrm{~h}$ at room temperature. The CAKI-1 and 786-O cell lines stably transfected with siPSME2 and/or siBNIP3 were trypsinized, centrifuged at $300 \mathrm{x}$ g for $5 \mathrm{~min}$ at room temperature, and resuspended with serum-free McCoy's 5a (Modified) medium and RPMI-1640, respectively. The CAKI-1 and 786-O cells transfected with siPSME2 were then treated with bafilomycin A1 (Baf-A1; $1 \mu \mathrm{mol} / \mathrm{l}$; Selleckchem) for $24 \mathrm{~h}$. The CAKI-1 cell line was transfected with both siPSME2 + siBNIP3 for $48 \mathrm{~h}$. A total of $1 \times 10^{5}$ cells $(200 \mu \mathrm{l})$ were added to the upper chamber and $600 \mu \mathrm{l}$ same medium with $10 \%$ FBS, was placed in the lower chamber in each well of a 24-well plate. After incubation at $37^{\circ} \mathrm{C}$ for $48 \mathrm{~h}$, the Transwell membrane was removed and a wet cotton swab was used to clear the cells from the upper chamber. Then, the cells that had migrated through the membrane were washed with $0.01 \mathrm{M}$ PBS and fixed with $4 \%$ paraformaldehyde at room temperature for $20 \mathrm{~min}$. Next, the cells were stained with $0.1 \%$ crystal violet (Beyotime Institute of Biotechnology) at room temperature for $10 \mathrm{~min}$ and images were captured using a phase contrast microscope (Nikon Corporation).

Cell proliferation assay. The CAKI-1 and 786-O cell lines transfected with siPSME2\#1/siPSME2\#2 or siNC, were seeded onto a 96-well plate, at a density of $3-5 \times 10^{3}$ cells $/ \mathrm{ml}$. The cell number was measured every $24 \mathrm{~h}$ using a CCK- 8 assay (MedChemExpress). Following incubation for $1.5 \mathrm{~h}$ at $37^{\circ} \mathrm{C}$ in a humidified incubator with $5 \% \mathrm{CO}_{2}$, the absorbance was measured at $450 \mathrm{~nm}$ using an automatic microplate spectrophotometer (Thermo Fisher Scientific, Inc.). The experiments were repeated at least three times.

Immunofluorescence (IF) assays. In total, $1 \times 10^{5}$ CAKI-1 and 786-O cell lines transfected with siPSME2\#2 or siNC were plated on coverslips, cultured overnight at $37^{\circ} \mathrm{C}$, then transfected with green fluorescent protein- microtubule-associated protein light chain3 plasmid (pGFP-LC3; Invitrogen; Thermo Fisher Scientific, Inc.) at a concentration of 1,000 virus particles/cell using Lipofectamine ${ }^{\circledR} 3000$ (Invitrogen; Thermo Fisher Scientific, Inc.) at room temperature. After transfection for $24 \mathrm{~h}$, the coverslips were fixed with $4 \%$ pro-cooled paraformaldehyde for $20 \mathrm{~min}$ at room temperature, then briefly incubated with DAPI (Invitrogen; Thermo Fisher Scientific, Inc.) at room temperature for $5 \mathrm{~min}$ in the dark. Finally, the slides were sealed with neutral balsam and viewed using a confocal fluorescence microscope (Axiovert $200 \mathrm{M}$; Zeiss GmbH). DAPI was used to label nuclei (blue), and autophagosomes were defined as GFP-LC3 puncta. The fluorescent puncta were analyzed using ImageJ v1.47 software (https://imagej.net). 
Transmission electron microscope (TEM). Following transfection with BNIP3 overexpression plasmid, the CAKI-1 and $786-\mathrm{O}$ cell lines were fixed with $2.5 \%$ paraformaldehyde in $100 \mathrm{mM}$ sodium phosphate buffer ( $\mathrm{pH}$ 7.4) at room temperature for $1 \mathrm{~h}$. Then, the cells were washed with PBS and fixed with $0.25 \%$ neutral glutaraldehyde overnight at $4^{\circ} \mathrm{C}$ for TEM sectioning. Subsequently, the sections were analyzed using a TEM (JEOL, Ltd.).

Western blot analysis. The cells, transfected with PSME2 siRNAs and BNIP3 overexpression plasmid, were harvested and lysed with RIPA (Beyotime Institute of Biotechnology). The protein concentration of each sample was measured using a Pierce ${ }^{\mathrm{TM}}$ Rapid Gold BCA Protein Assay kit (Thermo Fisher Scientific, Inc.) based on the manufacturer's guidelines. Total protein $(40 \mu \mathrm{g})$ was separated using $12.5 \%$ SDS-PAGE, transferred to PVDF membranes (MilliporeSigma), blocked with 5\% skimmed milk at room temperature for $2 \mathrm{~h}$, then incubated with the following primary antibodies: BNIP3 (cat. no. ab109362; 1:1,000 dilution; Abcam), PSME2 (cat. no. ab183727; 1:1,000 dilution; Abcam), LC3-I/II (cat.no. ABC929; 1:500 dilution; Sigma-Aldrich; Merck KgaA) sequestersome 1 (SQSTM1; cat. no. 18420-1-AP; 1:1,000 dilution; ProteinTech Group, Inc.) and GAPDH (cat. no. 60004-1-Ig; 1:10,000 dilution; ProteinTech Group, Inc.) on a shaker overnight at $4^{\circ} \mathrm{C}$. Following which, the membranes were washed with TBS containing $0.1 \%$ Tween-20 three times and incubated with HRP-conjugated secondary antibodies $(1: 10,000$ dilution; ProteinTech Group, Inc.) for $1 \mathrm{~h}$ at room temperature. The blotted proteins were observed using Immobilon ECL Ultra Western HRP Substrate (Merck KGaA), scanned with a Chemi-Doc System (Bio-Rad Laboratories, Inc.) and analyzed using ImageJ software (https://imagej.net).

$R T-q P C R$ and gene expression analysis. Following transfection with siPSME2 or BNIP3 overexpressing plasmid for $48 \mathrm{~h}$ in the 786-O and CAKI-1 cell lines, total RNA was extracted from the cells using TRIzol ${ }^{\circledR}$ (Invitrogen; Thermo Fisher Scientific, Inc.). Then, total RNA $(1 \mu \mathrm{g})$ was reverse transcribed at $37^{\circ} \mathrm{C}$ for $15 \mathrm{~min}$, then at $85^{\circ} \mathrm{C}$ for $5 \mathrm{sec}$ using a PrimeScript ${ }^{\mathrm{TM}}$ RT reagent kit, with gDNA Eraser (cat no. RR047A; Takara Bio Inc.). qPCR was performed using TB Green ${ }^{\circledR}$ Premix Ex Taq ${ }^{\mathrm{TM}}$ II (cat. no. RR820A; Takara Bio Inc.) according to the manufacturer's instructions on a LightCycler96 thermo cycler (Bio-Rad Laboratories, Inc.). The primer sequences were designed by TsingKe Biological Technology and the sequences are as follows: PSME2 forward, 5'-CTTTTCCAGGAGGCTGAGGAAT-3' and reverse, 5'-AGGGAAGTCAAGTCAGCCAC-3'; GAPDH forward, 5'-GGTGGTCTCCTCTGACTTCAACAG-3' and reverse, 5'-GTTGTTGTAGCCAAATTCGTTGT-3'; IL6 forward, 5'-TCCCCCTAGTTGTGTCTTGC-3' and reverse, 5'-GAAAAAGGCGGCTAGGTGTC-3'; TNF forward, 5'-CATCCAACCTTCCCAAACGC-3' and reverse, 5'-CGAAGTGGTGGTCTTGTTGC-3'; CXCL9 forward, 5'-ATTGGAGTGCAAGGAACCCC-3' and reverse, 5'-TAGTCCCTTGGTTGGTGCTG-3'; CXCL10 forward, 5'-TCCTGCAAGCCAATTTTGTCC-3' and reverse, 5'-TGTGGTCCATCCTTGGAAGC-3'; TNFSF13B (BLys) forward, 5'-GCAGACAGTGAAACACCAAC-3' and reverse,
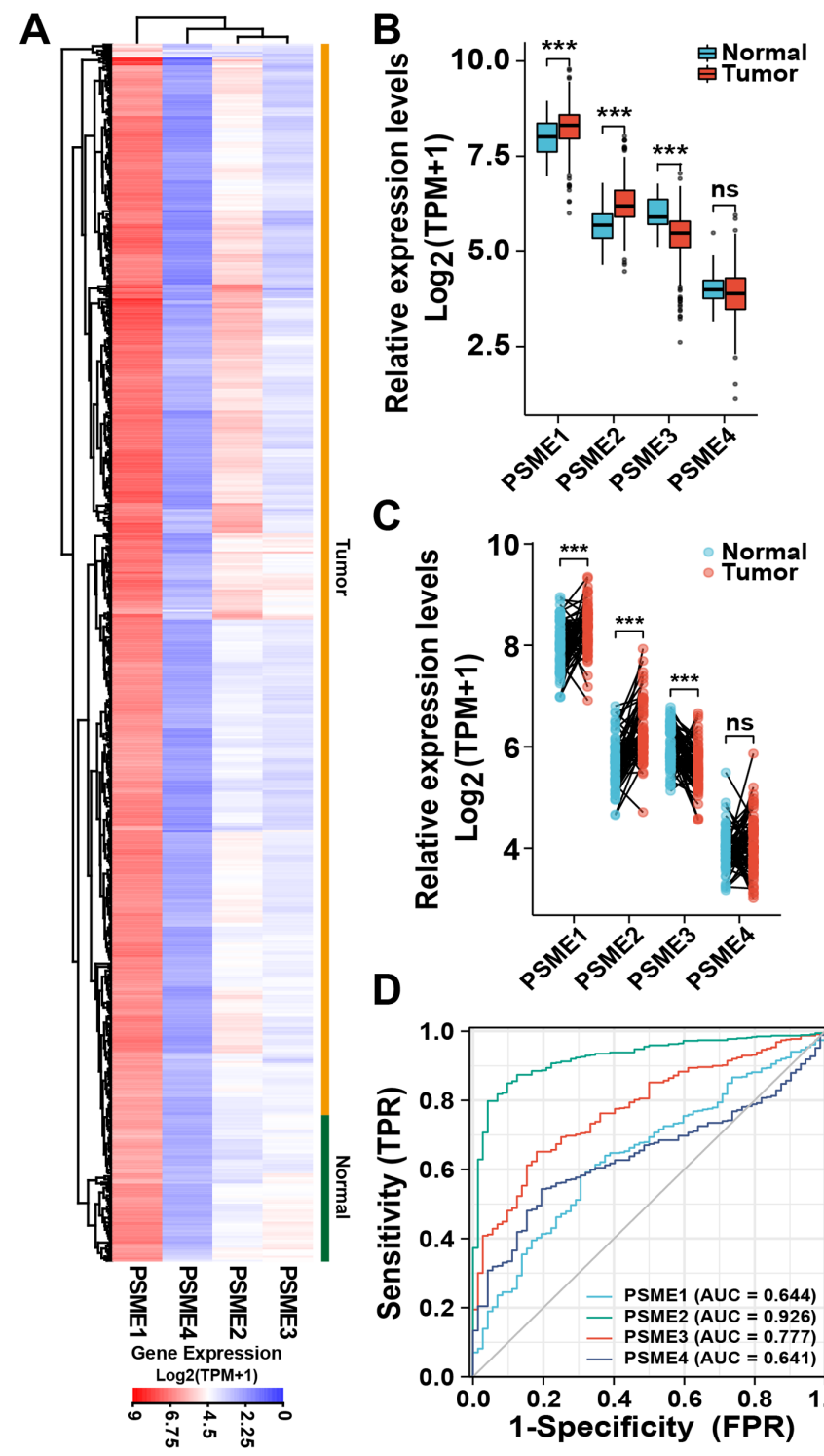

Figure 1. Expression profiles of four PSME genes in $\operatorname{KIRC}(\mathrm{n}=533$; yellow) and normal tissues ( $n=72$; green) samples from TCGA database. (A) Heatmap showing differential expression of the four PSME genes between the KIRC and in the normal. Red represents high expression, and blue indicates low expression. (B) Boxplots illustrating differential expression of the four PSME genes between normal and tumor tissues. (C) Expression of the four genes in normal adjacent and tumor tissues from patients with kidney cancer. The data were analyzed using paired t-tests. (D) Receiver operating characteristic curve analysis to assess the validity of the expression level from the four PSME genes in KIRC versus normal tissues. ${ }^{* * *} \mathrm{P}<0.001$. AUC, area under the ROC curve; TPR, true positive rate; FPR, false positive rate. PSME2, proteasome activator complex subunit 2 ; KIRC, kidney renal clear cell carcinoma.

5'-GATGTCCCATGGCGTAGGTC-3'. The following thermocycling conditions were used: Initial denaturation at $95^{\circ} \mathrm{C}$ for $30 \mathrm{sec}$ followed by 35 cycles at $95^{\circ} \mathrm{C}$ for $5 \mathrm{sec}$ and $60^{\circ} \mathrm{C}$ for $30 \mathrm{sec}$. Relative mRNA expression levels were calculated using the $2^{-\Delta \Delta C \mathrm{q}}$ method (45).

Statistical analysis. Statistical analysis was performed using GraphPad Prism (GraphPad Software, Inc.) or R v3.6.3 package (https://www.r-project.org/). The Mann-Whitney U test (Wilcoxon rank sum test) was used to compare two groups which did not meet normal distribution, including Figs. 1B and C, 2B, 4B and 5F. The Kruskal-Wallis test and 
A

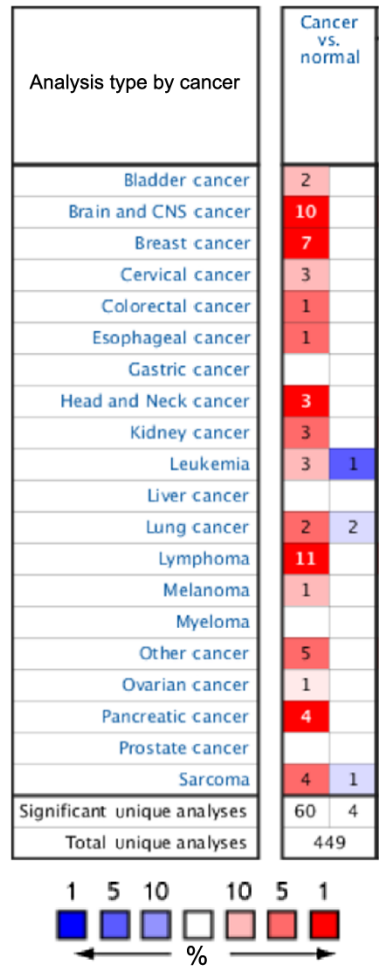

B

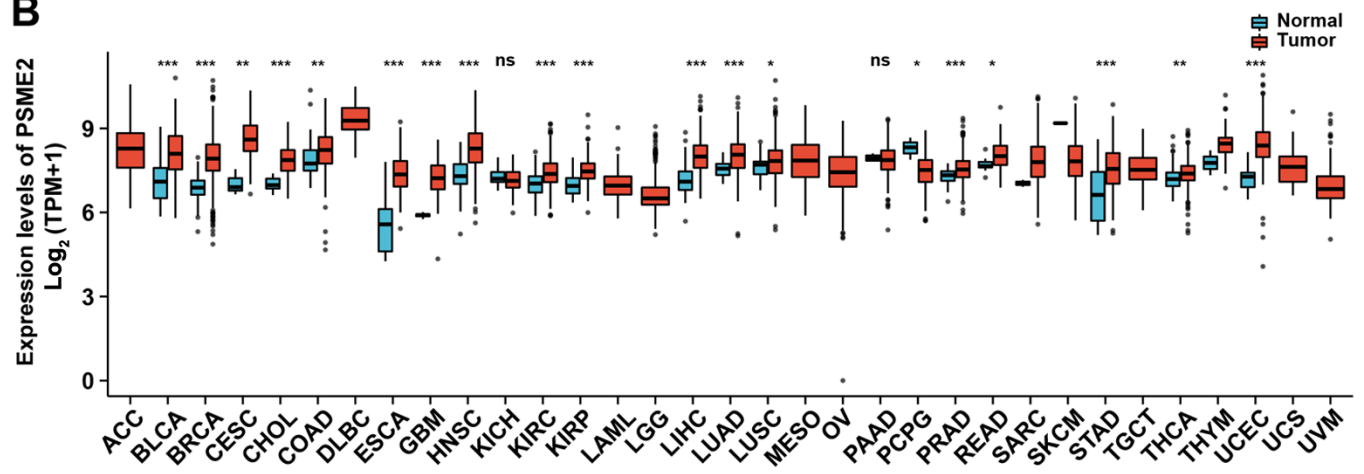

C

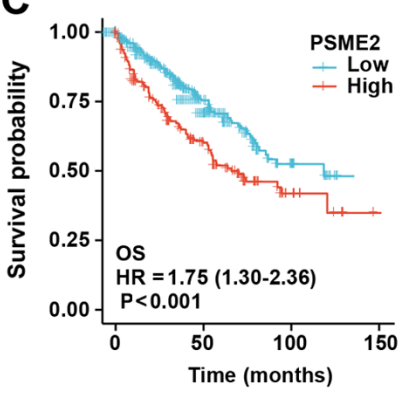

D

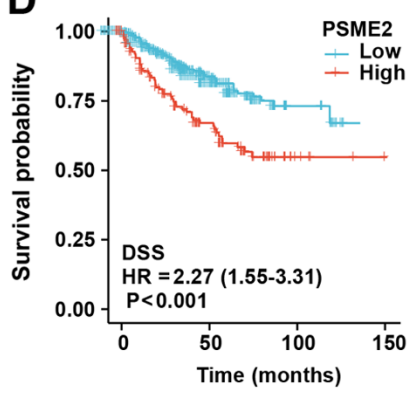

E

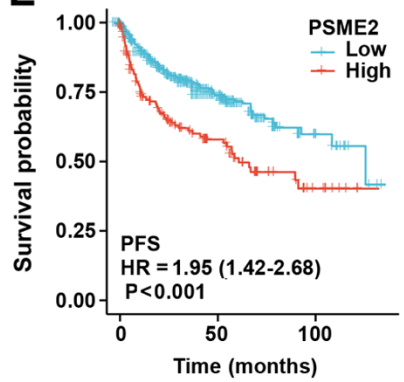

Figure 2. Expression profiling of PSME2 in diverse types of cancer and association of PSME2 expression with prognosis. (A) The PSME2 gene expression level in different types of cancer and normal tissues using the Oncomine database. The graph represents the datasets with statistically significant $(\mathrm{P}<0.01)$ overexpression (red) or lower expression (blue) in PSME2. (B) PSME2 differential expression level in 33 types of cancer $(\mathrm{n}=486)$ and normal ( $\mathrm{n}=338)$ tissues from The Cancer Genome Atlas database. ${ }^{*} \mathrm{P}<0.05,{ }^{* *} \mathrm{P}<0.01,{ }^{* * * *} \mathrm{P}<0.001$. PSME2 gene expression was associated with (C) OS, (D) DSS and (E) PFS time Kaplan-Meier survival analysis. HR, hazard ratio; OS, overall survival; DSS, disease-free survival; PFS, progression-free survival; ACC, adrenocortical carcinoma; BLCA, bladder urothelial carcinoma; BRCA, breast invasive carcinoma; CESC, cervical squamous cell carcinoma and endocervical adenocarcinoma; $\mathrm{CHOL}$, cholangiocarcinoma; COAD, colon adenocarcinoma; DLBC, lymphoid neoplasm diffuse large B-cell lymphoma; ESCA, esophageal carcinoma; GBM, glioblastoma multiforme; HNSC, head and neck squamous cell carcinoma; KICH, kidney chromophobe; KIRC, kidney renal clear cell carcinoma; KIRP, kidney renal papillary cell carcinoma; LAML, acute myeloid leukemia; LGG, brain lower grade glioma; LIHC, liver hepatocellular carcinoma; LUAD, lung adenocarcinoma; LUSC, lung squamous cell carcinoma; MESO, mesothelioma; OV, ovarian serous cystadenocarcinoma; PAAD, pancreatic adenocarcinoma; PCPG, pheochromocytoma and paraganglioma; PRAD, prostate adenocarcinoma; READ, rectum adenocarcinoma; SARC, sarcoma; SKCM, skin cutaneous melanoma; STAD, stomach adenocarcinoma; TGCT, testicular germ cell tumors; THCA, thyroid carcinoma; THYM, thymoma; UCEC, uterine corpus endometrial carcinoma; UCS, uterine carcinosarcoma; UVM, uveal melanoma; PSME2, proteasome activator complex subunit 2.

a Dwass-Steel-Critchlow-Fligner post hoc test was used to compare multiple groups, which did not meet normal distribution, including Figs. 5A and D, 6A and B, 7A and F and S1. Fisher's exact test was used to analyze the clinicopathological parameters of patients, including TNM stages. The Pearson's $\chi^{2}$ test was used to analyze differential PSME2 expression associated with the sex of the patients.

\section{Results}

PSME2 expression is upregulated in the KIRC dataset from $T C G A$. To determine the association between PSME2 mRNA expression and KIRC tissues, a transcriptional profile of PSME2, and other PSME genes, was analyzed using TCGA database. The data showed that, compared with that in normal tissues, PSME2 mRNA expression was upregulated in most tumor samples. Furthermore, among the PSME subtypes, PSME1 had a high level of expression. By contrast, the mRNA expression level of PSME4 was low and there was no difference in the expression level of PSME3 between the tumor and normal tissues (Fig. 1A). As shown in Fig. 1B, the median
mRNA expression levels of all the subtypes of PSME4 were significantly different between the tumor and normal tissues samples, except for PSME4. PSME1 and PSME2 were upregulated in the tumor tissues, whereas PSME3 was significantly downregulated. Paired tissue analysis also revealed that the expression levels of PSME2 were significantly upregulated $(\mathrm{P}<0.001)$ in the tumor samples compared with that in the adjacent normal samples (Fig. 1C). ROC curve analysis was used to investigate the validity of the mRNA expression level in the four PSME genes in KIRC and normal tissues. As shown in Fig. 1D, the AUCs of the four genes were all $>60 \%$. The gene with the highest AUC was PSME2 (92.6\%). Therefore, the mRNA expression level of PSME2 showed the best specificity and sensitivity to correctly distinguish tumor and adjacent normal kidney samples. Thus, PSME2 may play a critical role in kidney carcinoma.

Upregulated expression of PSME2 in cancer and the prognostic value of PSME2 in patients with ccRCC. To identify the function of PSME2 in different types of tumor, the expression levels between tumor and normal tissues were compared 


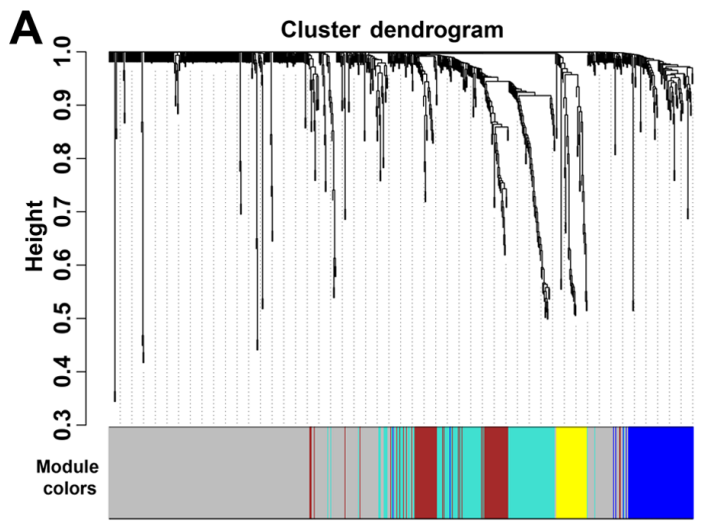

B
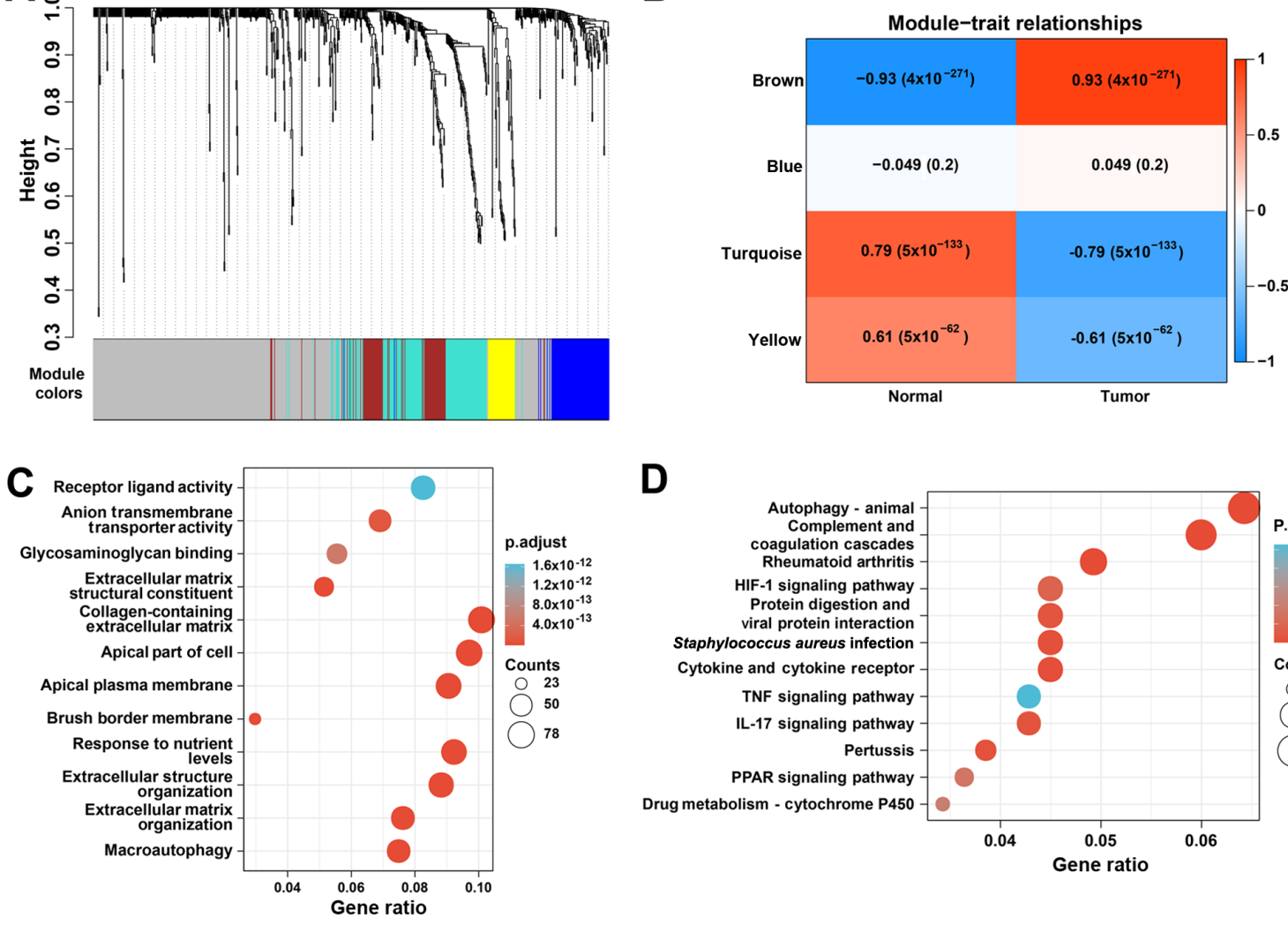

D

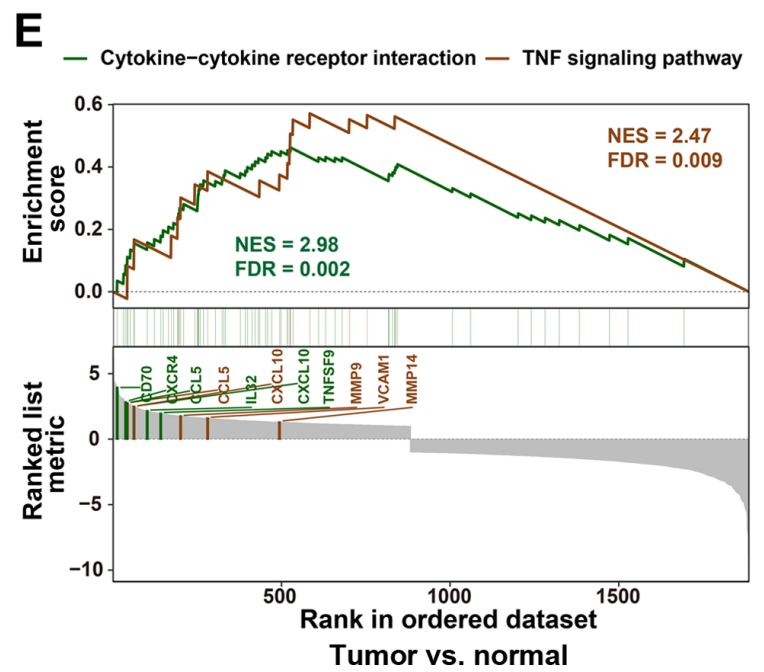

F - Autophagy - animal - Cytokine-cytokine receptor interaction - NF-kB signaling pathway

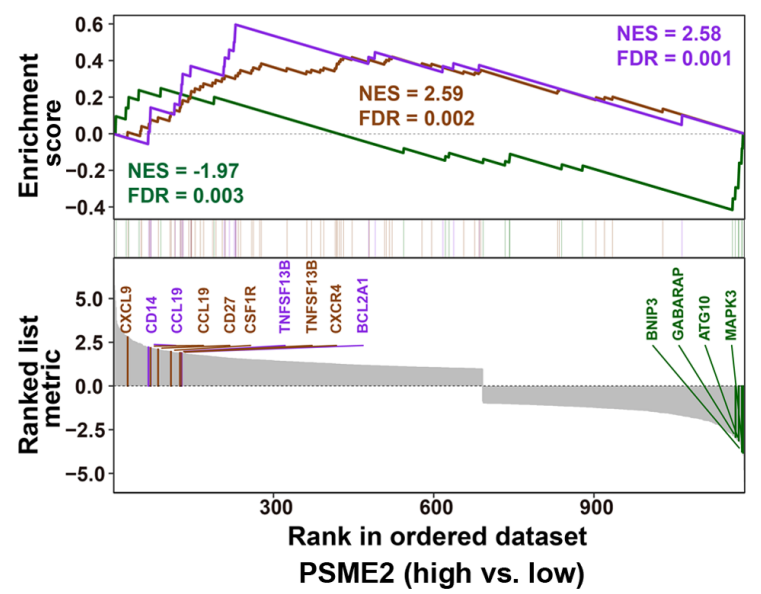

Figure 3. Functional characterization of PSME2 in ccRCC using WGCNA and GSEA. (A) WGCNA hierarchical cluster dendrogram of the top 25\% genes ranked by SD from large to small ccRCC. Each branch in the tree represents one gene. Each color represents one co-expression module. (B) PCC matrix of the correlation between MEs and clinicopathological status (normal and tumor) of ccRCC. Each row corresponds to a colored ME detected using WGCNA, and each column corresponds to a trait. The values of PCC ranged from -1 to 1 depending on the strength of the relationship. A positive value means that the probe sets in a specific co-expression module increase as the variable increases, while a negative value indicates a specific co-expression module decreases as the variable decreases. Each PCC value has the corresponding P-value in brackets. (C) GO enrichment analysis using The Database for Annotation, Visualization and Integrated Discovery. The horizontal axis represents the proportion of PSME2 enriched in each GO term. The vertical axis represents the annotation terms. The size of the bubble represents the number of genes in each GO term; depth of each circle color represents P-value. (D) KEGG enrichment for the differentially expressed genes in Fig. 3B. (E) GSEA revealing cytokine-cytokine receptor interaction and TNF signaling pathway associations between tumor and normal samples from TCGA. (F) GSEA revealing autophagy-animal, cytokine-cytokine receptor interaction and NF-kB signaling pathway associated with PSME2 expression levels from TCGA database. ME, module eigengenes; GO, Gene Ontology; KEGG, Kyoto Encyclopedia of Genes and Genomes; WGCNA, weighted gene co-expression network analysis; GSEA, Gene Set Enrichment Analysis; TCGA, The Cancer Genome Atlas; PSME2, proteasome activator complex subunit 2; ccRCC, clear cell renal cell carcinoma; NES, normalized enrichment score; FDR, false discovery rate; PCC, Pearson's correlation coefficient.

using the visualization tools in the Oncomine database. As illustrated in Fig. 2A, the Oncomine database contained 449 unique analyses for PSME2. It was found that PSME2 was upregulated in several types of cancers, including the brain and central nervous system cancer, breast cancer, lymphoma, and pancreatic cancer. A total of three studies revealed a significant increase in mRNA expression levels of PSME2 in kidney cancer samples compared with that in normal samples (46-48). 
A
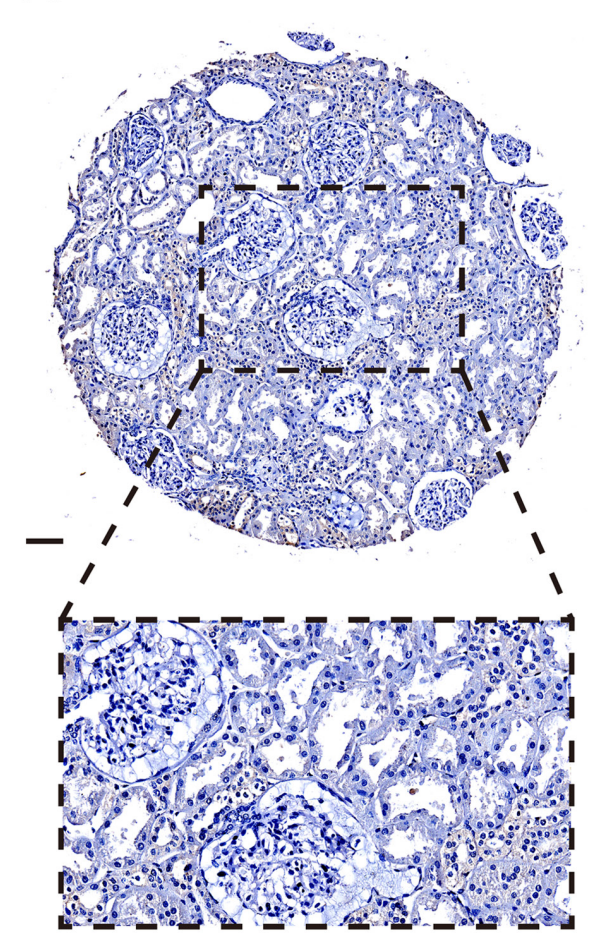

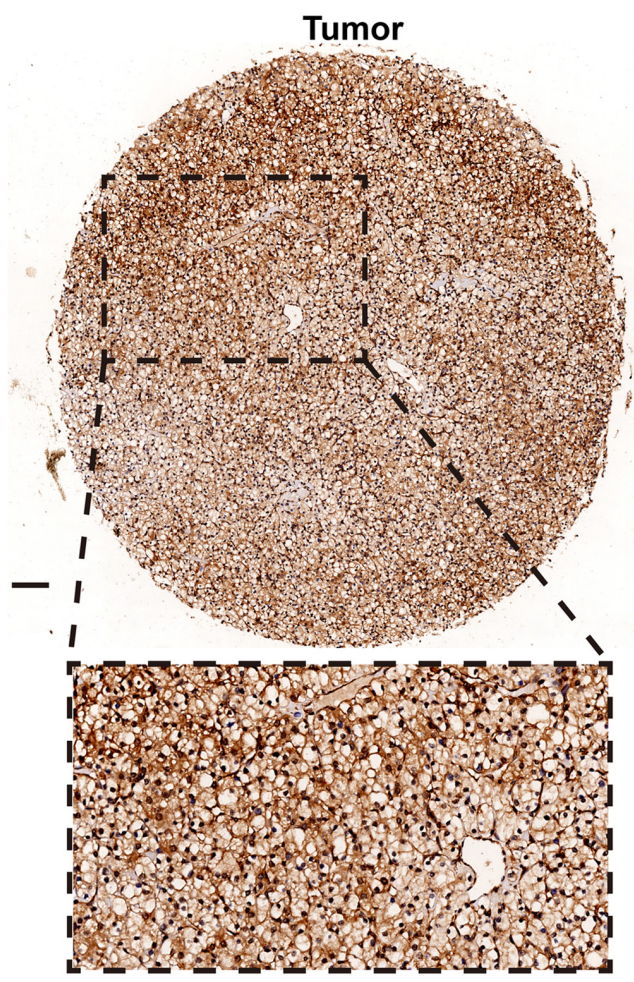

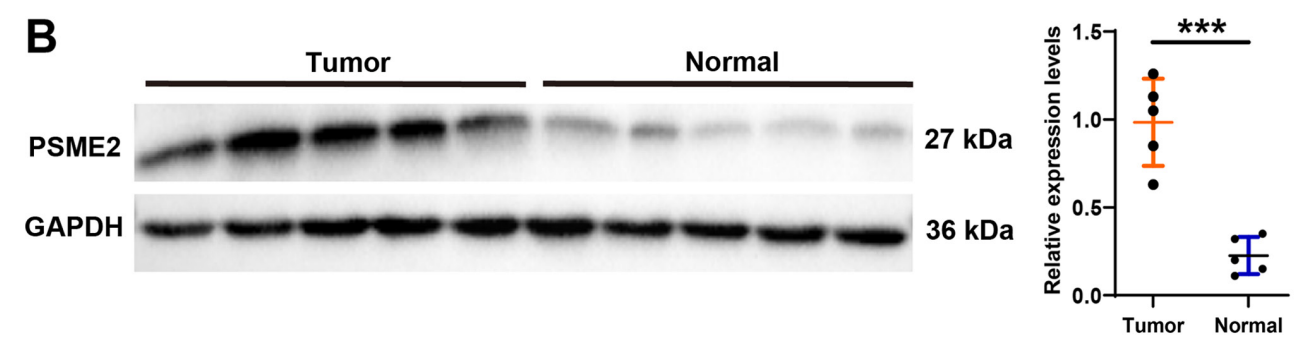

C
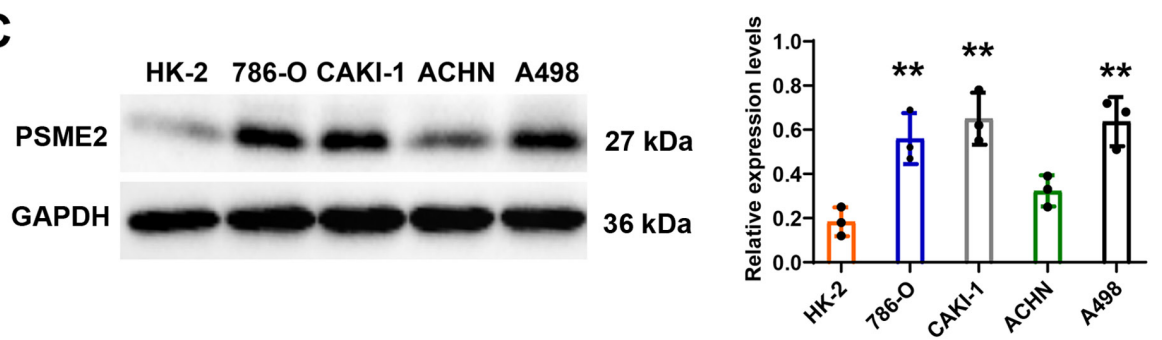

Figure 4. Expression levels of PSME2 in renal tumors, adjacent normal tissues, and renal cancer and normal cell lines. (A) Iummohistochemistry expression levels of PSME2 in cancer and adjacent normal tissues. Scale bar, $100 \mu \mathrm{m}$. Western blot analysis of PSME2 expression levels in (B) cancer and adjacent normal tissues, and (C) HK-2,786-O, CAKI-1, ACHN and A498 cell lines. PSME2, proteasome activator complex subunit $2 .{ }^{* *} \mathrm{P}<0.01 .{ }^{* * * *} \mathrm{P}<0.001$.

Accessing TCGA dataset, the PSME2 mRNA expression levels in various types of tumor tissues and adjacent normal tissues were compared (Fig. 2B). It was found that PSME2 was significantly upregulated in bladder urothelial carcinoma, breast invasive carcinoma, cholangiocarcinoma, cervical squamous cell carcinoma and endocervical adenocarcinoma, colon adenocarcinoma, esophageal carcinoma, glioblastoma multiforme, head and neck squamous cell carcinoma, KIRC, lung adenocarcinoma, liver hepatocellular carcinoma, lung squamous cell carcinoma, prostate adenocarcinoma, rectum adenocarcinoma, stomach adenocarcinoma, thyroid carcinoma and uterine corpus endometrial carcinoma, while it had lower expression levels in pheochromocytoma and paraganglioma compared with that in adjacent normal tissues. In addition, Kaplan-Meier plotter analysis for OS (Fig. 2C), DSS (Fig. 2D) and PFS (Fig. 2E) time revealed an association with PSME2 mRNA expression; therefore, PSME2 was significantly associated with patient prognosis. It was found that patients who had high mRNA expression level of PSME2, had a low survival rate. These findings indicated that PSME2 could be a tumor and prognosis-related marker.

Functional enrichment analysis of PSME2 mRNA expression level in ccRCC. To investigate the molecular mechanisms involved in ccRCC, WGCNA was performed and 194 differentially expressed probes among 12 tumor samples were divided 

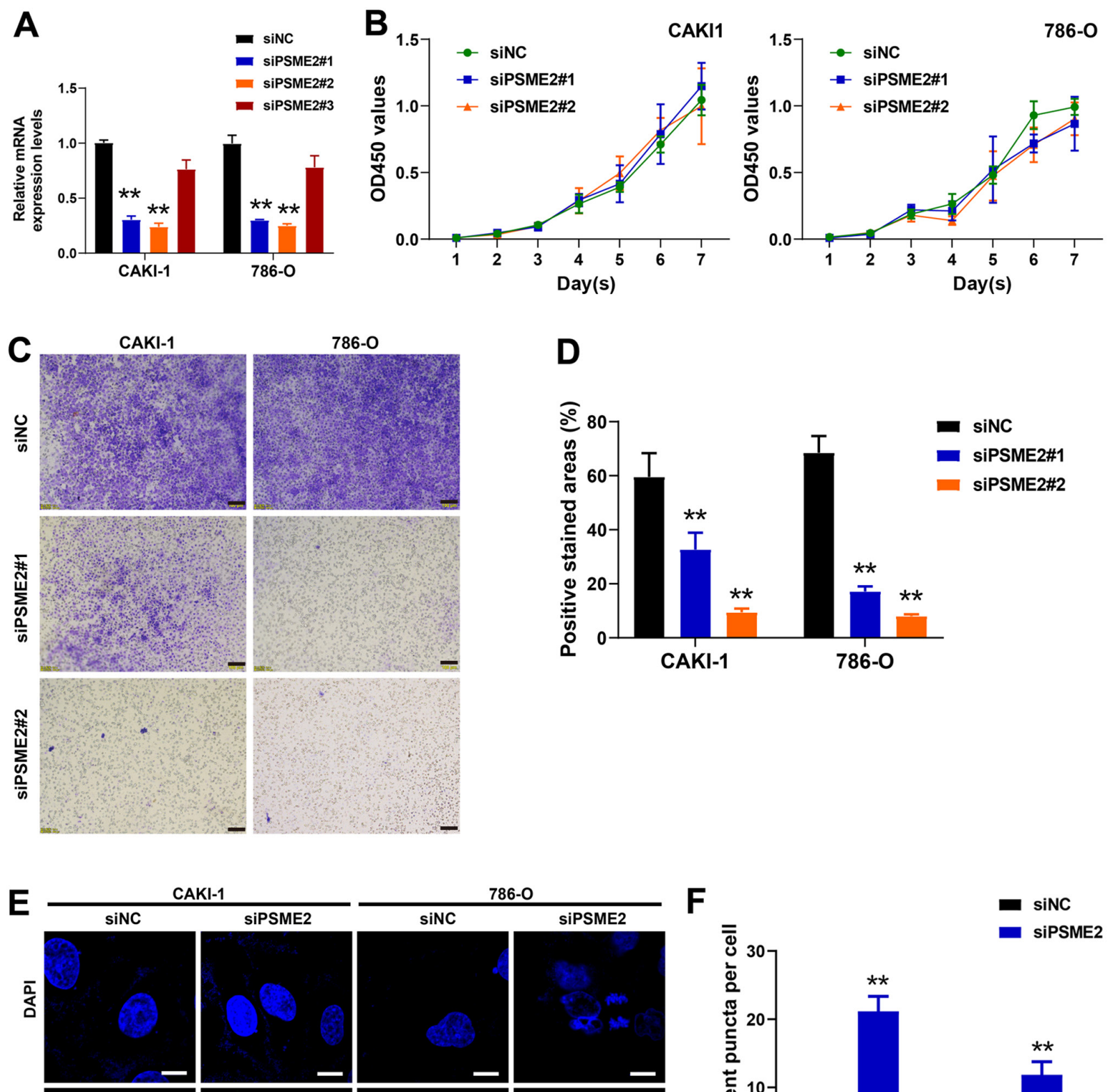

SiPSME2
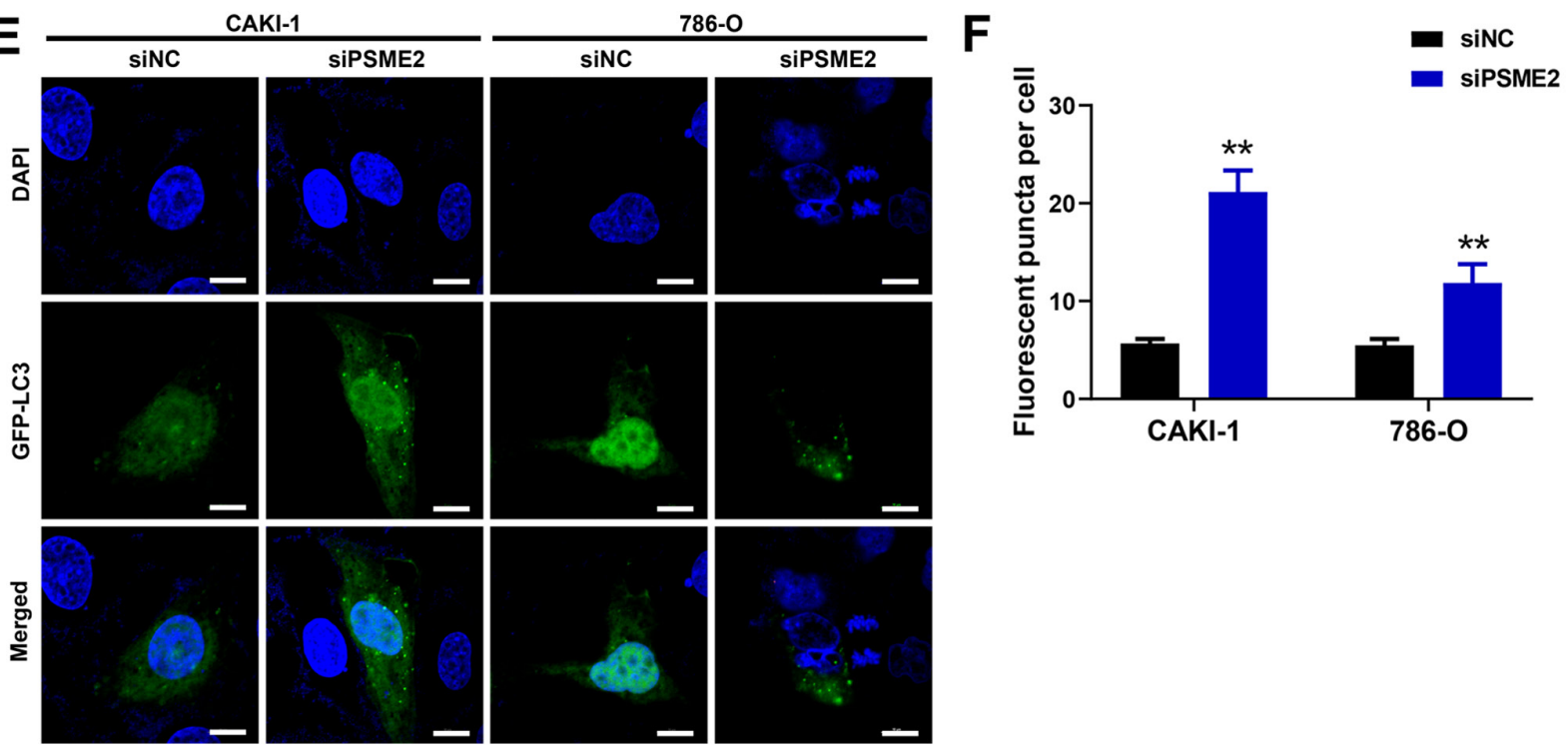

Figure 5. PSME2 promotes renal cancer cell invasion and inhibits autophagy. (A) mRNA expression levels of PSME2 were examined using reverse transcription-quantitative PCR. ${ }^{* *} \mathrm{P}<0.01$. (B) Cell proliferation of the CAKI-1 and 786-O cell lines transfected with siNC and siRNA was analyzed using a Cell Counting Kit-8 assay. (C) Transwell invasion assay was used to detect cell invasion following transfection with siRNA targeting PSME2. Scale bar, $100 \mu \mathrm{m}$. (D) The percent of positive stained areas in invaded cells per field. ${ }^{*} \mathrm{P}<0.01$. (E) Knockdown of PSME2 induces autophagy in the CAKI-1 and 786-O cell lines using confocal fluorescence microscopy. The cells were transfected with siPSME2\#2 and GFP-LC3 for $24 \mathrm{~h}$. Nuclei were labelled with DAPI (blue). Scale bar, $10 \mu \mathrm{m}$. (F) The fluorescent puncta per cell were quantified. ${ }^{* *} \mathrm{P}<0.01$. PSME2, proteasome activator complex subunit 2 ; si, small inhibiting; NC, negative control; GFP, green fluorescent protein.

into five-module colors of the top $25 \%$ genes ranked using cluster dendrogram trees, including the colors brown, blue, turquoise, yellow, and grey. The grey module was specified as the gene set that could not be assigned to any module and had no reference meaning (Fig. 3A). The association between module eigengenes and clinicopathological status (normal and 
A

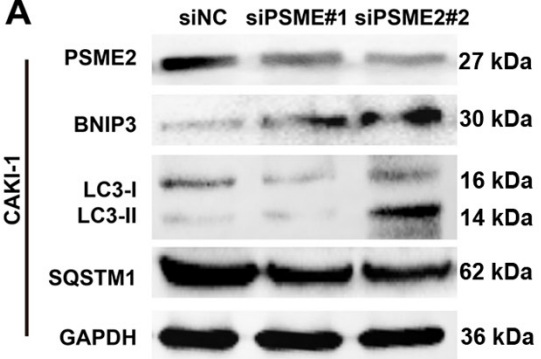

SINC SIPSME\#1 SIPSME2\#2

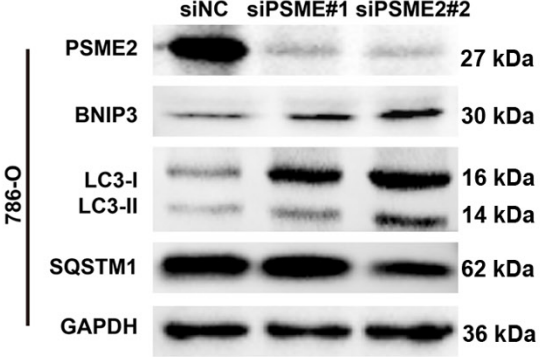

C
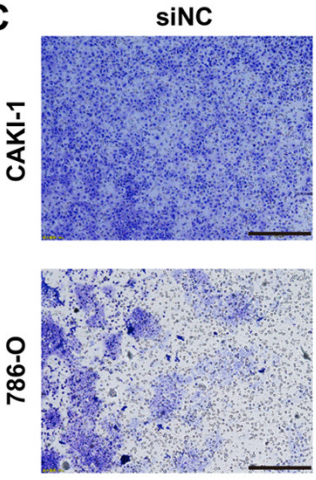

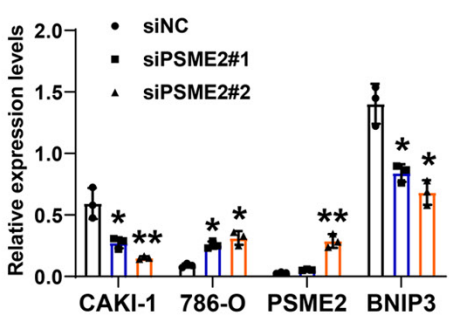

CAKI-1 786-O PSME2 BNIP3

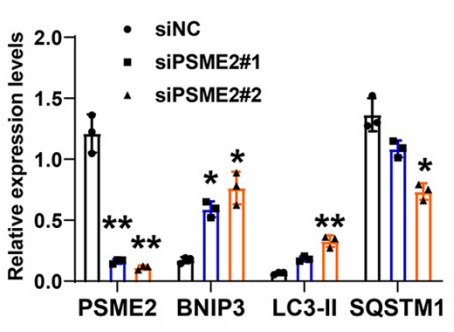

siPSME2 + Baf-A1
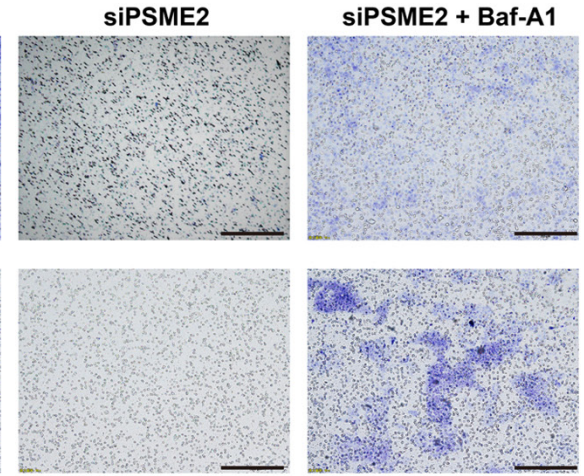

B

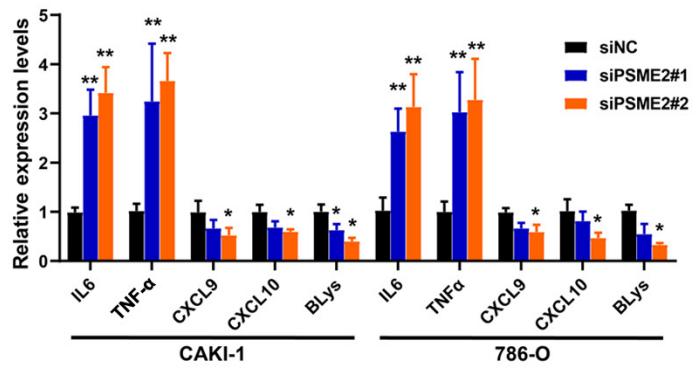

D

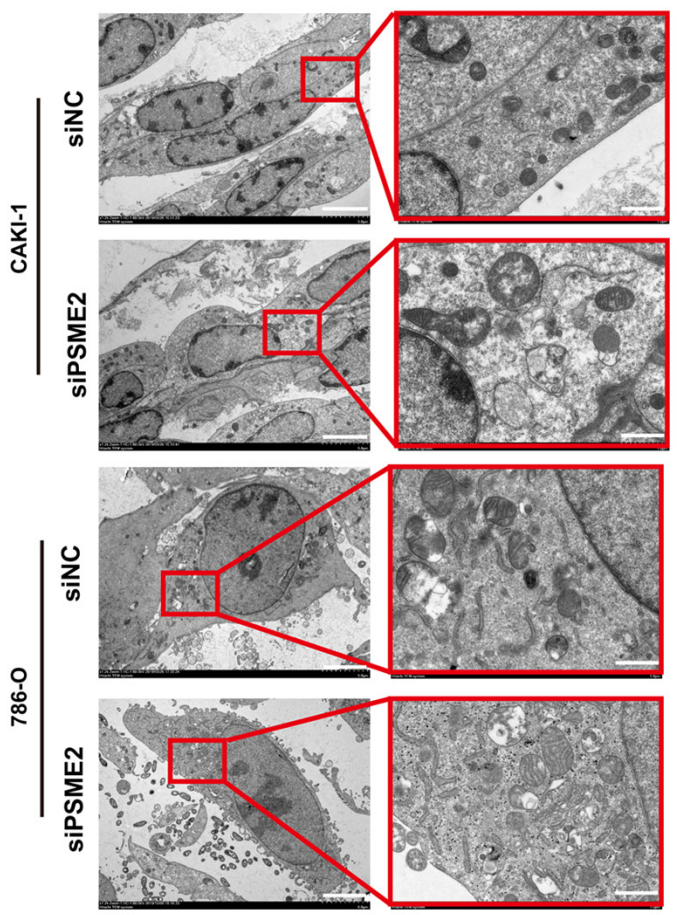

Figure 6. Effects of PSME2-knockdown on autophagy and tumor cytokine expression in the CAKI-1 and 786-O cell lines. (A) Autophagy-associated proteins were detected using western blot analysis and the results were quantified using densitometry. The data are presented as the mean \pm SD from three independent experiments. ${ }^{*} \mathrm{P}<0.05,{ }^{* *} \mathrm{P}<0.01$. (B) The mRNA expression level of IL-6, TNF- $\alpha$, CXCL9, CXCL10 and BLys were determined in the CAKI-1 and 786-O cell lines using reverse transcription-quantitative PCR. ${ }^{*} \mathrm{P}<0.05,{ }^{* *} \mathrm{P}<0.01$, compared to siNC control. (C) Cell invasion of CAKI-1 and $786-\mathrm{O}$ cell lines transfected with siPSME2 or siNC were analyzed following treatment with Baf-A1. Scale bar, $200 \mu \mathrm{m}$. (D) Transmission electron microscopy of autophagosomes in renal carcinoma cells transfected with siRNAs. Left scale bar, $5 \mu \mathrm{m}$; right scale bar, $1 \mu \mathrm{m}$. PSME2, proteasome activator complex subunit 2 ; si, small inhibiting; NC, negative control; Baf-A1, bafilomycin A1; SQSTM1, sequestersome 1; BNIP3, BCL2 interacting protein; CXCL, C-X-C motif chemokine ligand; BLys, B lymphocyte stimulator.

tumor) of ccRCC is shown in Fig. 3B. In Fig. 3B, the values of PCC ranged from -1 to 1 depending on the strength of the association. A positive value indicates the probe sets in a specific co-expression module increase as the variable increases, while a negative value indicates a specific co-expression module decreases as the variable decreases. Compared with that in the other modules, the genes in the turquoise module displayed the highest association with normal tissues. By contrast, the brown module showed the highest association with the tumor. Based on this method, the brown module, with the tumor, was identified as the clinically crucial module and was chosen for further investigation. Subsequently, the genes in the brown module in the tumor group were used for KEGG and GO enrichment analysis with DAVID. As depicted in Fig. 3C, in the brown module, most mRNAs were enriched in 'anion transmembrane transporter activity', 'extracellular matrix structural constituent', 'apical part of cell', 'collagen-containing extracellular matrix', 'response to nutrient levels', 'extracellular structure organization' and 'macroautophagy'. KEGG enrichment analysis demonstrated that genes were mainly enriched in 'autophagy-animal', 'complement and coagulation cascades', 'rheumatoid arthritis', 'protein digestion and viral protein interaction', 'Staphylococcus aureus infection', 'cytokine and cytokine receptor', 'IL-17 signaling pathway', and 'pertussis' (Fig. 3D). It was found that almost all the enriched functional terms and pathways were associated with the autophagy and cytokines.

To understand the pathways involved in PSME2 and ccRCC, GSEA analysis was conducted using the GSE dataset. The two most functional gene sets were enriched in the TNF signaling pathway, and cytokine and cytokine receptor interaction between the tumor and normal samples (Fig. 3E). Functional analysis of the PSME2 gene showed that several genes associated with numerous critical aspects of cancer were changed. The hub genes in cytokine and cytokine receptor interaction were CD70, CXCR4, CCL5, IL32, 

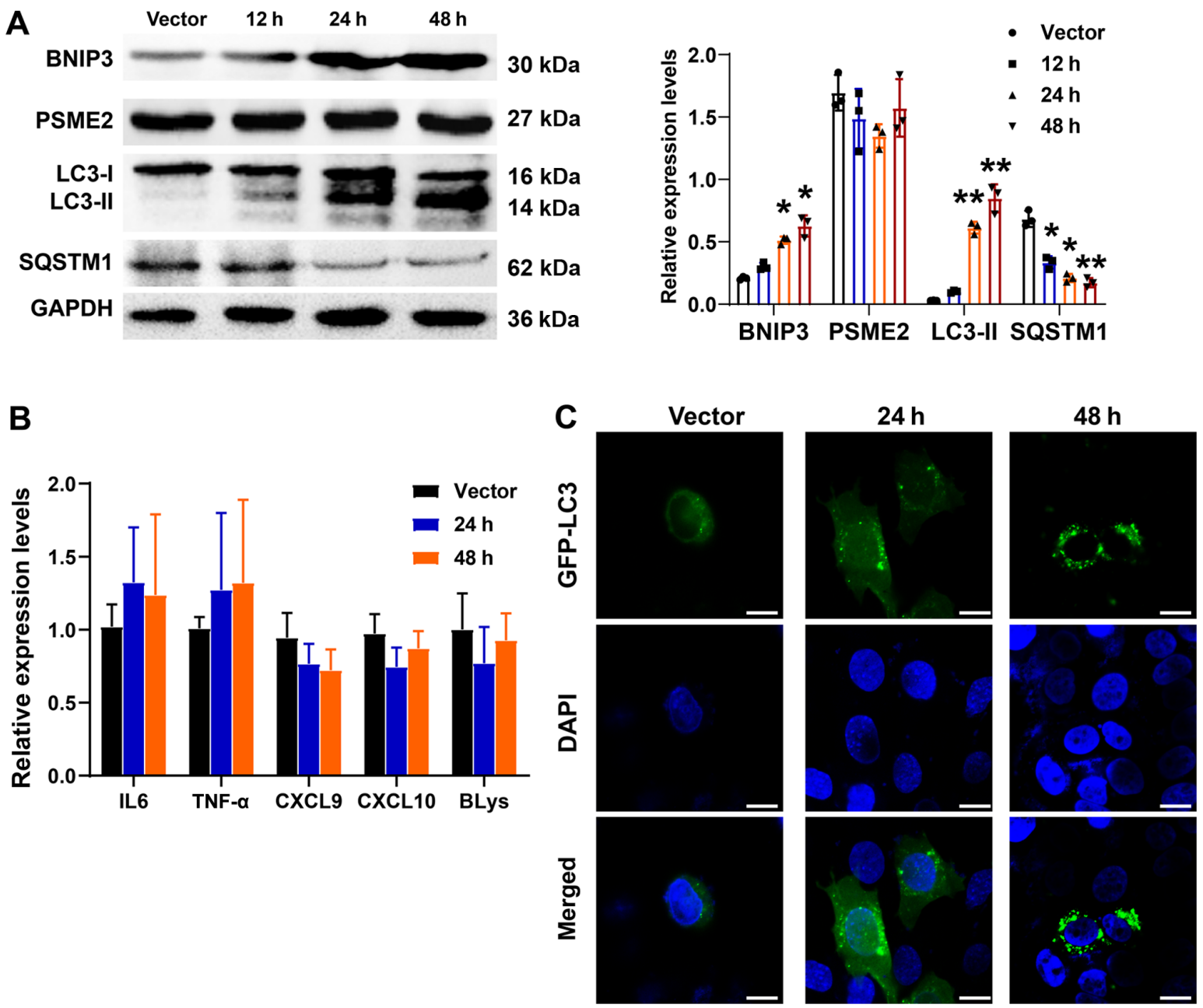

Figure 7. Continued.

CXCL10, and TNFSF9. In addition, the core regulators in the TNF signaling pathway included the CCL5, CXCL10, MMP9, VCAM1 and MMP14 genes. For patients with ccRCC and high expression levels of PSME2, the NF- $\mathrm{BB}$ signaling pathway and cytokine-cytokine receptor interaction were enriched in the two gene sets. CXCL9, CCL19, CD27, CSF1R, TNFSF13B and CXCR4, and CD14, CCL19, TNFSF13B and BCL2A1 were core regulators in the $\mathrm{NF}-\kappa \mathrm{B}$ signaling pathway and cytokine-cytokine receptor interaction, respectively. Whereas genes in the PSME2 low expression group were enriched in autophagy-animal. BNIP3, GABARAP, ATG10 and MAPK3 genes were found to be hub regulators (Fig. 3F).

Expression level of PSME2 in clinical samples and tumor cells. The bioinformatics results showed that PSME2 expression level was frequently upregulated in KIRC. To verify the results from bioinformatics analysis, the expression patterns of PSME2 were analyzed using IHC and its association with clinicopathological features in patients with kidney cancer was also investigated. Fig. 4A shows representative PSME2 staining images from the TMA. There was strong expression of PSME2 in the cytoplasm of the renal tumor tissues, but a weak expression level in adjacent normal tissues from patients with ccRCC. In addition, univariate analysis showed that $\mathrm{T}$ stage $(\mathrm{P}<0.001)$ and $\mathrm{M}$ stages $(\mathrm{P}<0.001)$ were significantly associated with PSME2 expression in the samples from patients with ccRCC. The details of the patient characteristics are listed in Table I. Western blot analysis also revealed that PSME2 protein expression level was more notable in tumor tissues compared with that in matched normal tissues (Fig. 4B). Then, the normal human kidney cell line (HK-2) and the renal carcinoma cell lines (786-O, CAKI-1, ACHN and A498) were used to determine the expression levels of PSME2. As shown in Fig. 4C, PSME2 was increased in the renal tumor cell lines, which was consistent with bioinformatics and IHC results.

Knockdown of PSME2 inhibits the invasive ability of the CAKI-1 and 786-O cell lines. To investigate the effect of PSME2 on cell proliferation and invasion, the 786-O and CAKI-1 cell lines were transfected with siPSME2 and siNC. After transfection for $48 \mathrm{~h}$, the cells were collected and the mRNA expression level of PSME2 was detected using RT-qPCR. As presented in Fig. 5A, after transfection with the 3 siRNAs targeting PSME2, the expression levels of the PSME2 gene in the cells transfected with siPSME2\#1 and siPSME2\#2 notably decreased compared with that in the control siRNA group $(\mathrm{P}<0.01)$. This confirmed that the siPSME2\#1 and siPSME2\#2 siRNAs were effective. Therefore, siPSME2\#1 and siPSME2\#2 were selected for further experimentation. The viability of the 786-O and CAKI-1 cell lines, detected 
D

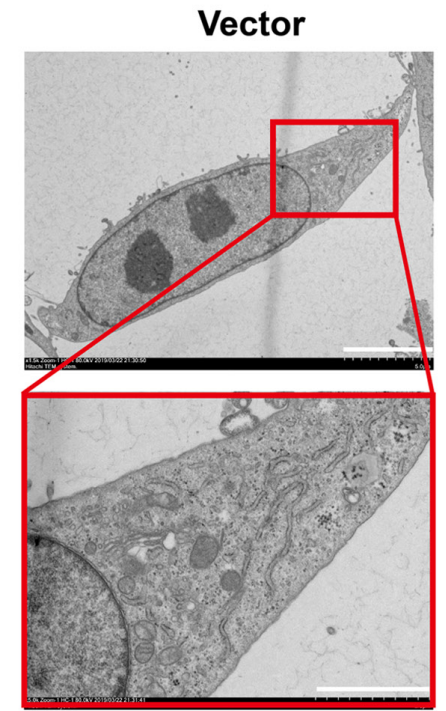

E
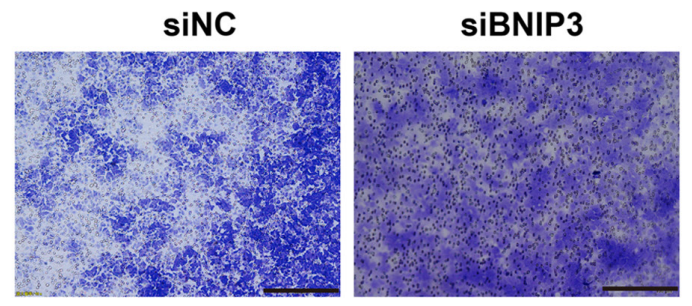

F

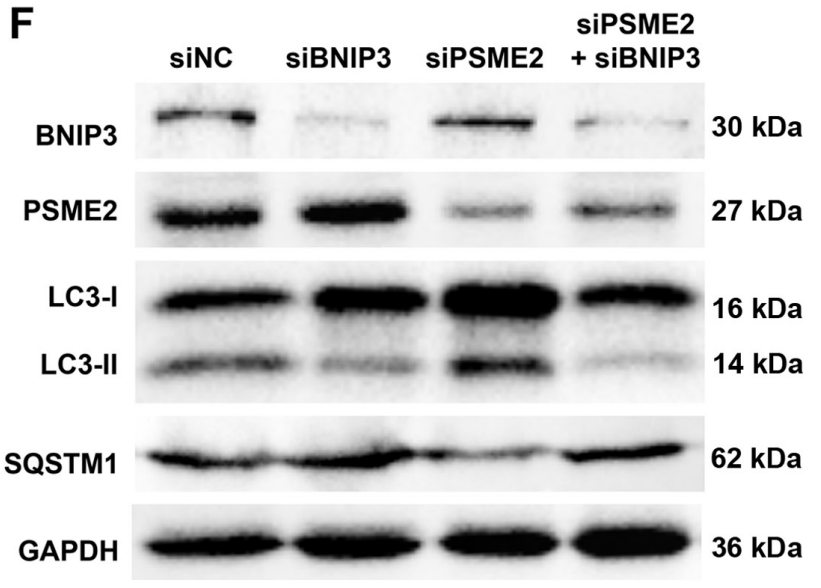

$24 \mathrm{~h}$

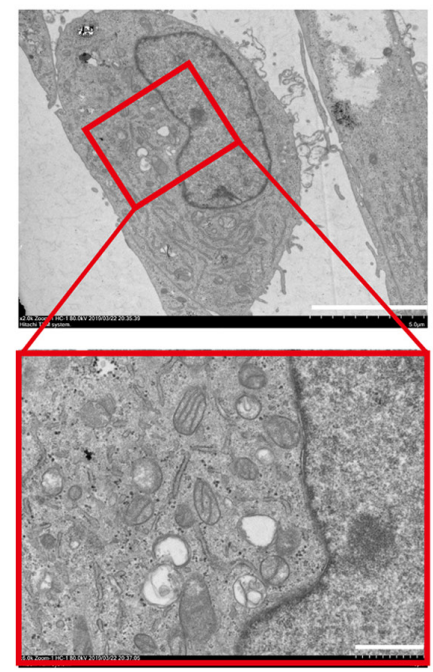

SIPSME2

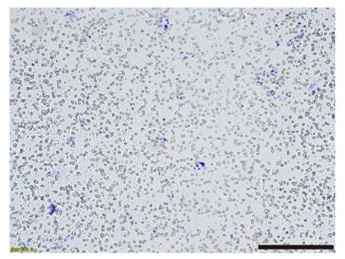

$48 \mathrm{~h}$

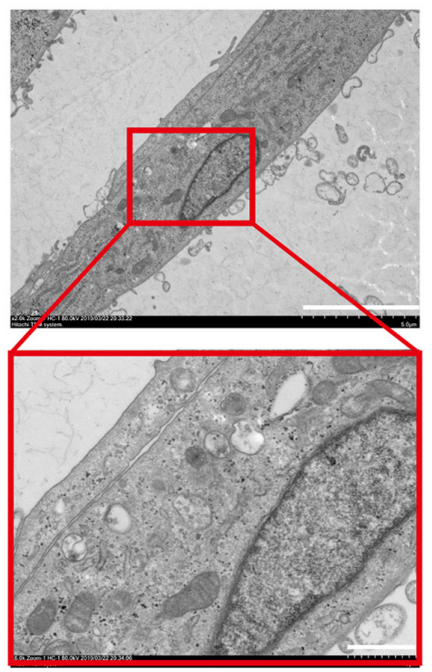

SIPSME2

+ siBNIP3

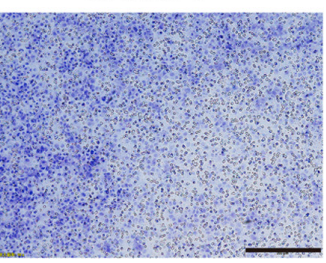

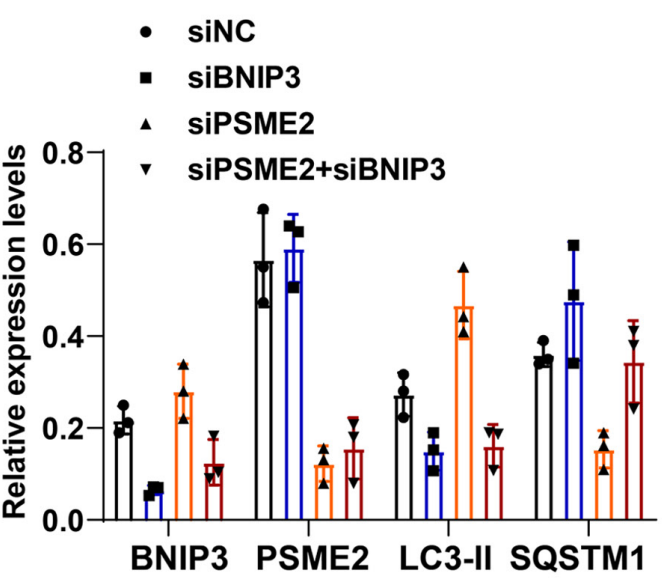

Figure 7. Effects of BNIP3 overexpression on autophagy and tumor cytokine expression level with or without siPSME2 in the CAKI-1 cell line. (A) Western blot analysis of autophagy-related proteins and PSME2 in empty vector and BNIP3-overexpressing cells at 12, 24 and $48 \mathrm{~h}$ following transfection, and the results were quantified using densitometry. ${ }^{*} \mathrm{P}<0.05,{ }^{* *} \mathrm{P}<0.01$, compared to vector. (B) IL-6, TNF- $\alpha$, CXCL9, CXCL10 and BLys mRNA expression levels were measured using reverse transcription-quantitative PCR. The cells were transfected with either empty plasmid or BNIP-3 overexpression vector for 24 and $48 \mathrm{~h}$. (C) Subcellular localizations of LC3 were determined using confocal microscopy. Scale bar, $10 \mu \mathrm{m}$. (D) Transmission electron microscope of the CAKI-1 cell line transfected with empty plasmid or BNIP-3 overexpression vector for 24 and $48 \mathrm{~h}$. Top scale bar, $5 \mu \mathrm{m}$; bottom scale bar, $1 \mu \mathrm{m}$. (E) Invasion of the CAKI-1 cell line simultaneously transfected with siBNIP3 and siPSME2 using a Transwell invasion assay. Scale bar, $200 \mu \mathrm{m}$. (F) A total of five protein biomarkers were found to be differentially expressed in BNIP3 or/and PSME2 knockout cells and the results were quantified using densitometry. PSME2, proteasome activator complex subunit 2; si, small inhibiting; NC, negative control; SQSTM1, sequestersome 1; BNIP3, BCL2 interacting protein; CXCL, C-X-C motif chemokine ligand; BLys, B lymphocyte stimulator.

using a CCK-8 assay, was not significantly different between the siPSME2\#1 and siPSME2\#2, and siNC groups (Fig. 5B). These data indicated that decreasing the expression level of PSME2 could not inhibit cell proliferation. The role of PSME2 in tumor cell invasion was detected using a Transwell invasion assay. Knockdown of PSME2 in the CAKI-1 and 786-O cell lines reduced invasion compared with that in the cells transfected with siNC (Fig. 5C). Quantitative analysis of the positive stained areas in the Transwell membrane showed that $\sim 35$ and $20 \%$ of the siPSME2\#1-transfected cells were invasive compared with those in the siNC-transfected CAKI-1 and 786-O cells, respectively. Furthermore, 10 and $8 \%$ of the cells in the siPSME2\#2 group were invasive in the CAKI-1 and 786-O cell lines $(\mathrm{P}<0.01$; Fig. 5D), which suggested that interference of PSME2 reduced the invasive abilities of the cancer cell lines. 
Table I. Univariate Cox regression analysis between low $(n=37)$ and high $(n=38)$ PSME2 expression groups and clinicopathological features in patients with clear cell renal cell carcinoma.

\begin{tabular}{lcc}
\hline & PSME2 expression & PSME2 expression \\
\cline { 2 - 3 } Characteristic & Low & High \\
\hline T stage, $\mathrm{n}(\%)$ & $22(29.3)$ & $17(22.7)$ \\
T1 & $5(6.7)$ & $5(6.7)$ \\
T2 & $10(13.3)$ & $15(20.0)$ \\
T3 & $0(0.0)$ & $1(1.3)$ \\
T4 & & $36(48.0)$ \\
N stage, n $(\%)$ & $33(44.0)$ & $2(2.7)$ \\
N0 & $4(5.3)$ & $29(38.7)$ \\
N1 & & $9(12.03)$ \\
M stage, $n(\%)$ & $34(45.3)$ & 0.149 \\
M0 & $3(4.0)$ & $26(34.6)$ \\
M1 & & $12(16.0)$ \\
Sex, $n(\%)$ & $23(30.6)$ & $63(54-67)$ \\
Male & $14(18.7)$ &
\end{tabular}

IQR, interquartile range; PSME2, proteasome activator complex subunit 2.

Inhibition of PSME2 promotes autophagy and affects the expression level of tumor cytokines in the CAKI-1 and 786-O cell lines. To gain insight into the role of PSME2 in autophagy, PSME2 expression was knocked down using siRNAs, then autophagosomes, which were stained with a specific GFP-LC3 in the 786-O and CAKI-1 cell lines, were analyzed (Fig. 5E and $\mathrm{F}$ ). The number of autophagosomes per cell was increased following siPSME2\#2 transfection, suggesting that knockdown of PSME2 induced autophagy in the CAKI-1 and 786-O cell lines. To determine the status of autophagy in the CAKI-1 and 786-O cell lines following knockdown of PSME2, the protein expression level of the autophagy markers, microtubule-associated protein 1 light chain 3 (LC3), which exists in two forms and transforms from the free form of LC3-I (18 kDa), to the smaller (16 kDa) proteolytic form, LC3-II during autophagy, BNIP3, and SQSTM1 was determined. From western blot analysis (Fig. 6A), LC3-I to LC3-II conversion was elevated and BNIP3 protein expression level was markedly increased in the CAKI-1 cell line. However, SQSTM1 was markedly decreased when the CAKI-1 cell line was transfected with siPSME2\#1 or siPSME2\#2. Similar results were found in the 786-O cell line. Subsequently, the mRNA expression level of tumor factors associated with immune cells was analyzed using RT-qPCR. IL- 6 and TNF- $\alpha$ mRNA expression levels were notably higher in the 786-O and CAKI-1 cell lines transfected siPSME2 compared with that in the siNC groups. On the contrary, CXCL9, CXCL10 and BLys mRNA expression levels were markedly decreased following transfection with siPSME2 (Fig. 6B). The cells transfected with siPSME2 were then treated with bafilomycin A1 (Baf-A1; an inhibitor of the autophagy) to inhibit autophagy activation. The results of the Transwell assay revealed that invasiveness was increased
(Fig. 6C). TEM was used to analyze the autophagosomes, to visualize the effect of siPSME2 on autophagy in the cancer cells. Autophagosomes were characterized by the vacuole-like structure of the bilayer-containing organelles in the cytoplasm. As shown in Fig. 6D, there were a few autophagosomes found in the control cells; however, they were abundant within the cytoplasm of the PSME2-knockout cells. These results indicated that PSME2 induced autophagy.

Effect of overexpression or knockdown of BNIP3 on autophagy and the expression level of tumor cytokines. To investigate the role of BNIP3 in the CAKI-1 cell line and the regulation of autophagy and tumor cytokines, a plasmid vector that overexpressed BNIP3 was constructed and its transfection efficiency was analyzed (Fig. S1). The protein expression levels of BNIP3, PSME2, LC3-I/II and SQSTM1, at 12, 24 and $48 \mathrm{~h}$ after transfection and the mRNA expression levels of IL-6, TNF- $\alpha$, CXCL9, CXCL10 and BLys were determined using western blot analysis and RT-qPCR, respectively. As shown in Fig. 7A, overexpression of BNIP3 caused LC3-I to LC3-II conversion and a decrease in SQSTM1 protein expression levels in a time-dependent manner. However, there was no significant difference in the protein expression level of PSME2. There were no noticeable changes in the mRNA expression levels for all the tumor cytokines following the overexpression of BNIP3 (Fig. 7B). To visualize autophagy, LC3 was detected following transfection with GFP-tagged proteins. Overexpression of BNIP3 induced a marked increase in the number of structures labeled by the autophagy marker, as shown in Fig. 7C. Similarly, overexpression of BNIP3 led to an increase in the number of autophagosomes in a time-dependent manner (Fig. 7D). Taken together, the data suggested that 
BNIP3 affected autophagy, but had no effect on the mRNA expression level of the tumor cytokines. The Transwell invasion experiments confirmed that knocking down BNIP3 promoted cell invasion, while low PSME2 expression inhibited cell invasion. With inhibition of these two proteins simultaneously, cell invasion was lower compared with that in the control group (Fig. 7E). Western blot analysis confirmed that BNIP3 knockdown suppressed autophagy-related protein expression level and inhibition of PSME2 expression promoted the expression of autophagy-related proteins. Nevertheless, suppressing both proteins, the autophagy inhibited by BNIP3 was restored, as shown in Fig. 7F.

\section{Discussion}

Renal cancer is one of the ten most challenging cancer types to diagnose and treat (49). Nearly $90 \%$ of kidney tumors are RCC and $80 \%$ of cases are ccRCC, making it the most dominant pathological subtype of $\operatorname{RCC}(50,51)$. Surgery is still the most effective treatment for local ccRCC; however, $30-35 \%$ of patients undergoing surgery will exhibit distant metastasis (50). The prognosis of ccRCC remains unsatisfactory, with a 5-year survival rate of $23 \%$ for advanced ccRCC, particularly for locally advanced and metastatic ccRCC (52). Therefore, it is important to investigate a biomarker, which is effective for the treatment and prognosis of ccRCC.

In previous studies, it was found that the role of PSME in tumors has been poorly reported, particularly in ccRCC. The present study described the discovery of aberrant PSME2 mRNA expression in human ccRCC tissues. PSME2 was upregulated in KIRC tissues using TCGA and Oncomine databases, suggesting that overexpression of PSME2 may promote ccRCC. Analysis of DSS, OS and PFS times demonstrated that high PSME2 expression level was predictive of poor perceived prognosis in patients with ccRCC. WGCNA was also used to determine co-expression networks of groups of genes from large expression data and four distinct co-expression modules were identified. The brown module was positively associated with the tumor tissue among the four modules. Furthermore, signal enrichment analysis of the differential genes in the brown module was used to identify GO terms and KEGG and pathways. The GO analysis results revealed the differentially expressed genes were enriched with 'anion transmembrane transporter activity', 'extracellular matrix structural constituent', 'apical part of the cell', 'collagen containing extracellular matrix', 'response to nutrient levels', 'extracellular structure organization', and 'macroautophagy'. These pathways were associated with tumorigenesis and development. The KEGG pathway analysis showed that these differential genes were associated with 'autophagy', 'complement and coagulation cascades', 'rheumatoid arthritis', 'protein digestion', 'viral protein interaction', 'Staphylococcus aureus infection', 'cytokine and cytokine receptor', 'IL-17 signaling pathway', and 'pertussis'. GSEA showed that PSME2 was associated with the TNF signaling pathway, cytokine-cytokine receptor interaction, autophagy-animal and the $\mathrm{NF}-\kappa \mathrm{B}$ signaling pathway in ccRCC. Using a comprehensive analysis of the association between WGCNA and GSEA, the findings indicated that the expression levels of the PSME2 in ccRCC may be involved in cytokine and cytokine receptor interaction and autophagy in the tumor tissue.
Next, the results from bioinformatics analysis were verified. The PSME2 protein expression level was highly expressed in ccRCC tissue, as confirmed using IHC staining and western blot analysis. A previous study reported that PSME2 was a biomarker of tumor invasion and metastasis (30). The cellular experiments then demonstrated that knockdown of PSME2 reduced the invasion of the ccRCC cell lines, but had no effect on cell proliferation. Subsequently, the molecular mechanisms associated with PSME2-induced invasiveness and metastasis of ccRCC were investigated, combined with bioinformatics analysis. Autophagy and tumor cytokine expression levels were investigated in the renal cancer cell lines transfected with or without PSME2-specific siRNA. LC3 is vital for the dynamic process of autophagosome formation. The characteristic signature of autophagic membranes is the conversion of LC3-I into LC3-II $(53,54)$. In addition, detecting LC3 using immunofluorescence has become a widely accepted method for identifying autophagy (55). Knockdown of PSME2 resulted in LC3-I conversion to LC3-II, which is located on pre-autophagosomes and autophagosomes, making it an autophagy marker (56). Inhibiting PSME2 can promote the formation of autophagosomes. Similarly, more autophagosomes, with GFP-LC3 fluorescence, were found in the siPSME2 group compared with that in the control group.

BNIP3 is a transmembrane protein, primarily located in the outer membrane of mitochondria. It competes with beclin-1 to bind to BCL2, releasing beclin-1 and inducing autophagy $(57,58)$. It was found that the knockdown of PSME2 increased BNIP3 protein expression levels in the 786-O and CAKI-1 cell lines. This indicated that inhibiting PSME2 promoted autophagy. SQSTM1 is a scaffold protein in autophagosomes (59) and a stress-inducible protein (60), with multiple domains that mediate its communications with different binding molecules, including a TNF- associated receptor-6 binding domain (61), a Phox1 and Bem1p domain (62), a ZZ-type zinc finger domain, a Keap1-interacting domain, an LC3-interacting domain, and an ubiquitin-associated domain $(60,63,64)$. SQSTM1 is a principle selective autophagy receptor and an important protein in the autophagic clearance of polyubiquitinated proteins $(65,66)$. SQSTM1 binds to the ubiquitinated protein in the autophagosome and fuses with the lysosome to form the autophagosome to be cleared. Likewise, decreased expression of SQSTM1 indicated inhibition of PSME2-induced autophagy. Taken together, we hypothesized that renal cancer cells enhance invasion and inhibit autophagy by overexpressing PSME2.

IL-6 is a tumor cytokine associated with mortality. High mRNA expression levels of IL-6 were associated with decreased survival time in patients with ccRCC $(67,68)$. TNF- $\alpha$ is a core adjustor of a complex cytokine network, which not only mediates the pro-inflammatory response, but also regulates the interaction between cells, cell differentiation and cell death (69). A high amount of evidence has indicated that TNF- $\alpha$ has tumor promoting activity (70-73). CXCL9 and CXCL10 are IFN-inducible CXCR3 ligands, and key regulators in recruiting $\mathrm{T}$ cells to the tumor microenvironment. It has been reported that patients with ccRCC and a high expression level of CXCL9 and CXCL10 have poor survival times, and are more likely to have early recurrence $(74,75)$. 
BLys is a member of the TNF superfamily of ligands and is constitutively expressed on the cell membrane of macrophages, monocytes, activated T cells, dendritic cells, neutrophils, and antigen-presenting cells (76). High BLys protein expression in malignant tumors, including B-cell non-multiple myeloma, Hodgkin's lymphoma, Hodgkin's lymphoma and chronic lympho-cytic leukemia, has been reported $(77,78)$. It is noteworthy that IL6 and TNF- $\alpha$ expression levels were increased, but CXCL9/10 and BLys were significantly decreased in ccRCC cell lines transfected with PSME2 siRNA compared with that in the NC siRNA group. However, when BNIP3 was overexpressed, the mRNA expression levels of the five cytokines did not change. It was found that reduced PSME2 expression augments the mRNA expression level of inflammatory factors (IL- 6 and TNF- $\alpha$ ) and attenuates CXCL9/10 and BLys associated with the tumor microenvironment.

In summary, the findings from the present study demonstrated that PSME2 was upregulated in ccRCC tissues compared with that in normal tissues. This overexpression was not associated with cell proliferation, while it may be associated with cell invasion, autophagy and the tumor microenvironment. The expression of BNIP3 was found via the use of siPSME2 and the effect of BNIP3overexpression on PSME2 was also found. In addition, the preliminary function of PSME2 in ccRCC was identified using rescue and knockdown experiments. To the best of our knowledge, the present study is the first report the potential significance and function of PSME2 in ccRCC. Future investigations into the molecular mechanism of ccRCC should concentrate on PSME2 and the tumor microenvironment of ccRCC. A limitation to the current study was that only an association between PSME2 and ccRCC was found, additional experiments are required to validate the results. The results provide further information on the effect of PSME2 and the aggressiveness of tumor cells via cytokine and immune cells in ccRCC. In addition, PSME2 could be considered as a biomarker and therapeutic target for ccRCC.

\section{Acknowledgements}

Not applicable.

\section{Funding}

This study was supported by grants from the National Natural Science Foundation of China (grant no. 21772131) and the Fundamental Research Funds for the Science and Technology Department of Sichuan Province (grant nos. 2019YFSY0004 and 2019YFS0298).

\section{Availability of data and materials}

The datasets used and/or analyzed in the current study are available from the corresponding author upon reasonable request.

\section{Authors' contributions}

$\mathrm{XW}, \mathrm{FW}, \mathrm{GH}, \mathrm{XL}$ designed the research and wrote the manuscript. XW, YD, JC, YZ preformed the bioinformatics analysis and cell experiments. XW and GH analyzed the data and confirmed the authenticity of all the raw data generated during the study. XW, FW, GH and XL contributed to the critical reading and correction of the manuscript. All authors have read and approved the final manuscript.

\section{Ethics approval and consent to participate}

Ethics committee approval was obtained from the Institutional Ethics Committee of West China Hospital of Sichuan University (approval no. 2021211A) to the commencement of the study laid down in the 1964 Declaration of Helsinki. Written informed consent was provided by all the participants.

\section{Patient consent for publication}

Not applicable.

\section{Competing interests}

The authors declare that they have no competing interests.

\section{References}

1. Ahn K, Erlander M, Leturcq D, Peterson PA, Früh K and Yang Y: In vivo characterization of the proteasome regulator PA28. J Biol Chem 271: 18237-18242, 1996.

2. Coux O, Tanaka K and Goldberg AL: Structure and functions of the 20S and 26S proteasomes. Annu Rev Biochem 65: 801-847, 1996.

3. Ma CP, Willy PJ, Slaughter CA and DeMartino GN: PA28, an activator of the $20 \mathrm{~S}$ proteasome, is inactivated by proteolytic modification at its carboxyl terminus. J Biol Chem 268: 22514-22519, 1993.

4. Kuroda $\mathrm{K}$ and Liu $\mathrm{H}$ : The proteasome inhibitor, bortezomib, induces prostate cancer cell death by suppressing the expression of prostate-specific membrane antigen, as well as androgen receptor. Int J Oncol 54: 1357-1366, 2019.

5. Rechsteiner M and Hill CP: Mobilizing the proteolytic machine: Cell biological roles of proteasome activators and inhibitors. Trends Cell Biol 15: 27-33, 2005.

6. Song X, von Kampen J, Slaughter CA and DeMartino GN: Relative functions of the alpha and beta subunits of the proteasome activator, PA28. J Biol Chem 272: 27994-28000, 1997.

7. Wang YF, Yu M, te Pas MFW, Yerle M, Liu B, Fan B, Xiong TA and Li K: Sequence characterization, polymorphism and chromosomal localizations of the porcine PSME1 and PSME2 genes. Anim Genet 35: 361-366, 2004.

8. McCusker D, Wilson M and Trowsdale J: Organization of the genes encoding the human proteasome activators PA28alpha and beta. Immunogenetics 49: 438-445, 1999.

9. Zaiss DMW and Kloetzel PM: A second gene encoding the mouse proteasome activator PA28beta subunit is part of a LINE1 element and is driven by a LINE1 promoter. J Mol Biol 287: 829-835, 1999.

10. Kuehn L and Dahlmann B: Structural and functional properties of proteasome activator PA28. Mol Biol Rep 24: 89-93, 1997.

11. Demartino GN and Gillette TG: Proteasomes: Machines for all reasons. Cell 129: 659-662, 2007.

12. Lee J, An S, Jung JH, Kim K, Kim JY, An IS and Bae S: MUL1 E3 ligase regulates the antitumor effects of metformin in chemoresistant ovarian cancer cells via AKT degradation. Int J Oncol 54: 1833-1842, 2019.

13. Kandil E, Kohda K, Ishibashi T, Tanaka K and Kasahara M: PA28 subunits of the mouse proteasome: Primary structures and chromosomal localization of the genes. Immunogenetics 46: 337-344, 1997.

14. Wang Q, Pan F, Li S, Huang R, Wang X, Wang S, Liao X, Li D and Zhang L: The prognostic value of the proteasome activator subunit gene family in skin cutaneous melanoma. J Cancer 10: 2205-2219, 2019 
15. Feng J, Xiao T, Lu SS, Hung XP, Yi H, He QY, Huang W, Tang YY and Xiao ZQ: ANXA1 derived peptides suppress gastric and colon cancer cell growth by targeting EphA2 degradation. Int J Oncol 57: 1203-1213, 2020.

16. Wójcik C, Tanaka K, Paweletz N, Naab U and Wilk S: Proteasome activator (PA28) subunits, alpha, beta and gamma (Ki antigen) in NT2 neuronal precursor cells and HeLa S3 cells. Eur J Cell Biol 77: 151-160, 1998.

17. Khor B, Bredemeyer AL, Huang CY, Turnbull IR, Evans R, Maggi LB Jr, White JM, Walker LM, Carnes K, Hess RA, et al: Proteasome activator PA200 is required for normal spermatogenesis. Mol Cell Biol 26: 2999-3007, 2006.

18. Noda C, Tanahashi N, Shimbara N, Hendil KB and Tanaka K Tissue distribution of constitutive proteasomes, immunoproteasomes, and PA28 in rats. Biochem Biophys Res Commun 277: $348-354,2000$

19. Huang L, Haratake K, Miyahara H and Chiba T: Proteasome activators, PA28 gamma and PA200, play indispensable roles in male fertility. Sci Rep 6: 9, 2016.

20. Kaymaz Y, Oduor CI, Yu H, Otieno JA, Ong'echa JM, Moormann AM and Bailey JA: Comprehensive Transcriptome and Mutational Profiling of Endemic Burkitt Lymphoma Reveals EBV Type-Specific Differences. Mol Cancer Res 15: 563-576, 2017.

21. Tanahashi N, Yokota K, Ahn JY, Chung CH, Fujiwara T, Takahashi E, DeMartino GN, Slaughter CA, Toyonaga T, Yamamura K, et al: Molecular properties of the proteasome activator PA28 family proteins and gamma-interferon regulation. Genes Cells 2: 195-211, 1997.

22. Minor MM, Hollinger FB, McNees AL, Jung SY, Jain A, Hyser JM, Bissig KD and Slagle BL: Hepatitis B Virus HBx Protein Mediates the Degradation of Host Restriction Factors through the Cullin 4 DDB1 E3 Ubiquitin Ligase Complex. Cells 9: 9, 2020.

23. Li J, Powell SR and Wang X: Enhancement of proteasome function by PA28\&alpha; overexpression protects against oxidative stress. FASEB J 25: 883-893, 2011.

24. Grune T, Catalgol B, Licht A, Ermak G, Pickering AM, Ngo JK and Davies KJ: HSP70 mediates dissociation and reassociation of the $26 \mathrm{~S}$ proteasome during adaptation to oxidative stress. Free Radic Biol Med 51: 1355-1364, 2011.

25. Adelöf J, Andersson M, Porritt M, Petersen A, Zetterberg M, Wiseman J and Hernebring M: PA28 $\alpha \beta$ overexpression enhances learning and memory of female mice without inducing $20 \mathrm{~S}$ proteasome activity. BMC Neurosci 19: 70, 2018.

26. Miyagi T, Tatsumi T, Takehara T, Kanto T, Kuzushita N, Sugimoto Y, Jinushi M, Kasahara A, Sasaki Y, Hori M, et al: Impaired expression of proteasome subunits and human leukocyte antigens class I in human colon cancer cells. J Gastroenterol Hepatol 18: 32-40, 2003.

27. Cerruti F, Martano M, Petterino C, Bollo E, Morello E, Bruno R, Buracco P and Cascio P: Enhanced expression of interferon-gamma-induced antigen-processing machinery components in a spontaneously occurring cancer. Neoplasia 9 : 960-969, 2007.

28. Ebert MPA, Krüger S, Fogeron ML, Lamer S, Chen J, Pross M, Schulz HU, Lage H, Heim S, Roessner A, et al: Overexpression of cathepsin B in gastric cancer identified by proteome analysis. Proteomics 5: 1693-1704, 2005.

29. Perroud B, Lee J, Valkova N, Dhirapong A, Lin PY, Fiehn O, Kültz D and Weiss RH: Pathway analysis of kidney cancer using proteomics and metabolic profiling. Mol Cancer 5: 64, 2006.

30. Milioli HH, Santos Sousa K, Kaviski R, Dos Santos Oliveira NC, De Andrade Urban C, De Lima RS, Cavalli IJ and De Souza Fonseca Ribeiro EM: Comparative proteomics of primary breast carcinomas and lymph node metastases outlining markers of tumor invasion. Cancer Genomics Proteomics 12: 89-101, 2015.

31. Huang Q, Huang Q, Chen W, Wang L, Lin W, Lin J and Lin X: Identification of transgelin as a potential novel biomarker for gastric adenocarcinoma based on proteomics technology. J Cancer Res Clin Oncol 134: 1219-1227, 2008.

32. Huang Q, Huang Q, Lin W, Lin J and Lin X: Potential roles for PA28beta in gastric adenocarcinoma development and diagnosis J Cancer Res Clin Oncol 136: 1275-1282, 2010.

33. Zheng D-L, Huang Q-L, Zhou F, Huang Q-J, Lin J-Y and Lin X PA28 $\beta$ regulates cell invasion of gastric cancer via modulating the expression of chloride intracellular channel 1. J Cell Biochem 113: 1537-1546, 2012.

34. Kim JE, Koo KH, Kim YH, Sohn J and Park YG: Identification of potential lung cancer biomarkers using an in vitro carcinogenesis model. Exp Mol Med 40: 709-720, 2008.
35. Chen JY, Xu L, Fang WM, Han JY, Wang K and Zhu KS: Identification of PA28 $\beta$ as a potential novel biomarker in human esophageal squamous cell carcinoma. Tumour Biol 39: $1010428317719780,2017$.

36. Rhodes DR, Yu J, Shanker K, Deshpande N, Varambally R, Ghosh D, Barrette T, Pandey A and Chinnaiyan AM: ONCOMINE: A cancer microarray database and integrated data-mining platform. Neoplasia 6: 1-6, 2004.

37. Langfelder $\mathrm{P}$ and Horvath S: WGCNA: An R package for weighted correlation network analysis. BMC Bioinformatics 9: 559, 2008.

38. Langfelder P and Horvath S: Eigengene networks for studying the relationships between co-expression modules. BMC Syst Biol 1: 54, 2007.

39. Huang W, Sherman BT and Lempicki RA: Systematic and integrative analysis of large gene lists using DAVID bioinformatics resources. Nat Protoc 4: 44-57, 2009.

40. Huang W, Sherman BT and Lempicki RA: Bioinformatics enrichment tools: Paths toward the comprehensive functional analysis of large gene lists. Nucleic Acids Res 37: 1-13, 2009.

41. Azhar RA, de Castro Abreu AL, Broxham E, Sherrod A, Ma Y, Cai J, Gill TS, Desai M and Gill IS: Histological analysis of the kidney tumor-parenchyma interface. J Urol 193: 415-422, 2015.

42. Moch H, Artibani W, Delahunt B, Ficarra V, Knuechel R, Montorsi F, Patard JJ, Stief CG, Sulser T and Wild PJ: Reassessing the current UICC/AJCC TNM staging for renal cell carcinoma. Eur Urol 56: 636-643, 2009.

43. Margulis V, McDonald M, Tamboli P, Swanson DA and Wood CG: Predictors of oncological outcome after resection of locally recurrent renal cell carcinoma. J Urol 181: 2044-2051, 2009.

44. Kuang P, Deng H, Liu H, Cui H, Fang J, Zuo Z, Deng J, Li Y, Wang $X$ and Zhao L: Sodium fluoride induces splenocyte autophagy via the mammalian targets of rapamycin (mTOR) signaling pathway in growing mice. Aging (Albany NY) 10: $1649-1665,2018$

45. Livak KJ and Schmittgen TD: Analysis of relative gene expression data using real-time quantitative PCR and the 2(-Delta Delta C(T)) Method. Methods 25: 402-408, 2001

46. Jones J, Otu H, Spentzos D, Kolia S, Inan M, Beecken WD, Fellbaum C, Gu X, Joseph M, Pantuck AJ, et al: Gene signatures of progression and metastasis in renal cell cancer. Clin Cancer Res 11: 5730-5739, 2005.

47. Yusenko MV, Kuiper RP, Boethe T, Ljungberg B, van Kessel AG and Kovacs G: High-resolution DNA copy number and gene expression analyses distinguish chromophobe renal cell carcinomas and renal oncocytomas. BMC Cancer 9: 152, 2009.

48. Yusenko MV, Zubakov D and Kovacs G: Gene expression profiling of chromophobe renal cell carcinomas and renal oncocytomas by Affymetrix GeneChip using pooled and individual tumours. Int J Biol Sci 5: 517-527, 2009.

49. Zhu WK, Xu WH, Wang J, Huang YQ, Abudurexiti M, Qu YY, Zhu YP, Zhang HL and Ye DW: Decreased SPTLC1 expression predicts worse outcomes in ccRCC patients. J Cell Biochem 121: $1552-1562,2020$.

50. Hu J, Chen Z, Bao L, Zhou L, Hou Y, Liu L, Xiong M, Zhang Y, Wang B, Tao Z, et al: Single-Cell Transcriptome Analysis Reveals Intratumoral Heterogeneity in ccRCC, which Results in Different Clinical Outcomes. Mol Ther 28: 1658-1672, 2020.

51. Reustle A, Di Marco M, Meyerhoff C, Nelde A, Walz JS, Winter S, Kandabarau S, Büttner F, Haag M, Backert L, et al: Integrative -omics and HLA-ligandomics analysis to identify novel drug targets for ccRCC immunotherapy. Genome Med 12: 32, 2020.

52. Wan B, Liu B, Huang Y and Lv C: Identification of genes of prognostic value in the ccRCC microenvironment from TCGA database. Mol Genet Genomic Med 8: e1159, 2020.

53. Dikic I and Elazar Z: Mechanism and medical implications of mammalian autophagy. Nat Rev Mol Cell Biol 19: 349-364, 2018.

54. Tanida I, Ueno T and Kominami E: LC3 conjugation system in mammalian autophagy. Int J Biochem Cell Biol 36: 2503-2518, 2004.

55. Tanida I, Ueno T and Kominami E: LC3 and Autophagy. Methods Mol Biol 445: 77-88, 2008

56. Kabeya Y, Mizushima N, Ueno T, Yamamoto A, Kirisako T, Noda T, Kominami E, Ohsumi Y and Yoshimori T: LC3, a mammalian homologue of yeast Apg8p, is localized in autophagosome membranes after processing. EMBO J 19: 5720-5728, 2000 . 
57. Li L, Tan J, Miao Y, Lei P and Zhang Q: ROS and Autophagy: Interactions and Molecular Regulatory Mechanisms. Cell Mol Neurobiol 35: 615-621, 2015.

58. Zhang J, Zhang C, Jiang X, Li L, Zhang D, Tang D, Yan T, Zhang Q, Yuan H, Jia J, et al: Involvement of autophagy in hypoxia-BNIP3 signaling to promote epidermal keratinocyte migration. Cell Death Dis 10: 234, 2019.

59. Christian F, Krause E, Houslay MD and Baillie GS: PKA phosphorylation of $\mathrm{p} 62 / \mathrm{SQSTM} 1$ regulates PB1 domain interaction partner binding. Biochim Biophys Acta 1843: 2765-2774, 2014

60. Katsuragi Y, Ichimura Y and Komatsu M: p62/SQSTM1 functions as a signaling hub and an autophagy adaptor. FEBS J 282: 4672-4678, 2015.

61. Wooten MW, Geetha T, Seibenhener ML, Babu JR, Diaz-Meco MT and Moscat J: The p62 scaffold regulates nerve growth factor-induced NF-kappaB activation by influencing TRAF6 polyubiquitination. J Biol Chem 280: 35625-35629, 2005.

62. Lamark T, Perander M, Outzen H, Kristiansen K, Øvervatn A, Michaelsen E, Bjørkøy G and Johansen T: Interaction codes within the family of mammalian Phox and Bemlp domain-containing proteins. J Biol Chem 278: 34568-34581, 2003.

63. Lin X, Li S, Zhao Y, Ma X, Zhang K, He X and Wang Z: Interaction domains of p62: A bridge between p62 and selective autophagy. DNA Cell Biol 32: 220-227, 2013.

64. Islam MA, Sooro MA and Zhang P: Autophagic Regulation of p62 is Critical for Cancer Therapy. Int J Mol Sci 19: 15, 2018.

65. Johansen $\mathrm{T}$ and Lamark T: Selective autophagy mediated by autophagic adapter proteins. Autophagy 7: 279-296, 2011.

66. Zhang Y, Mun SR, Linares JF, Towers CG, Thorburn A, Diaz-Meco MT, Kwon YT and Kutateladze TG: Mechanistic insight into the regulation of SQSTM1/p62. Autophagy 15: 735-737, 2019.

67. Kays JK, Koniaris LG, Cooper CA, Pili R, Jiang G, Liu Y and Zimmers TA: The Combination of Low Skeletal Muscle Mass and High Tumor Interleukin-6 Associates with Decreased Survival in Clear Cell Renal Cell Carcinoma. Cancers (Basel) 12: 12, 2020.

68. Liu T, Xia Q, Zhang H, Wang Z, Yang W, Gu X, Hou T, Chen Y, Pei X, Zhu G, et al: CCL5-dependent mast cell infiltration into the tumor microenvironment in clear cell renal cell carcinoma patients. Aging (Albany NY) 12: 21809-21836, 2020.
69. Xu WH, Shi SN, Xu Y, Wang J, Wang HK, Cao DL, Shi GH, Qu YY, Zhang HL and Ye DW: Prognostic implications of Aquaporin 9 expression in clear cell renal cell carcinoma. J Transl Med 17: 363, 2019.

70. Mikami S, Mizuno R, Kosaka T, Saya H, Oya M and Okada Y: Expression of TNF- $\alpha$ and CD44 is implicated in poor prognosis, cancer cell invasion, metastasis and resistance to the sunitinib treatment in clear cell renal cell carcinomas. Int J Cancer 136: 1504-1514, 2015

71. Leibovich SJ, Polverini PJ, Shepard HM, Wiseman DM, Shively V and Nuseir N: Macrophage-induced angiogenesis is mediated by tumour necrosis factor-alpha. Nature 329: 630-632, 1987.

72. Michalaki V, Syrigos K, Charles P and Waxman J: Serum levels of IL-6 and TNF-alpha correlate with clinicopathological features and patient survival in patients with prostate cancer. $\mathrm{Br} \mathrm{J}$ Cancer 90: 2312-2316, 2004.

73. Balkwill F: Tumour necrosis factor and cancer. Nat Rev Cancer 9: 361-371, 2009.

74. Liu W, Liu Y, Fu Q, Zhou L, Chang Y, Xu L, Zhang W and Xu J: Elevated expression of IFN-inducible CXCR3 ligands predicts poor prognosis in patients with non-metastatic clear-cell renal cell carcinoma. Oncotarget 7: 13976-13983, 2016.

75. Choueiri TK, Atkins MB, Rose TL, Alter RS, Ju Y, Niland K, Wang Y, Arbeit R, Parasuraman S, Gan L, et al: A phase 1b trial of the CXCR4 inhibitor mavorixafor and nivolumab in advanced renal cell carcinoma patients with no prior response to nivolumab monotherapy. Invest New Drugs 39: 1019-1027, 2021.

76. Jiang P, Yueguo W, Huiming H, Hongxiang Y, Mei W and Ju S: B-Lymphocyte stimulator: A new biomarker for multiple myeloma. Eur J Haematol 82: 267-276, 2009.

77. Nardelli B, Moore PA, Li Y and Hilbert DM: B lymphocyte stimulator (BLyS): A therapeutic trichotomy for the treatment of B lymphocyte diseases. Leuk Lymphoma 43: 1367-1373, 2002.

78. Shivakumar L and Ansell S: Targeting B-lymphocyte stimulator/B-cell activating factor and a proliferation-inducing ligand in hematologic malignancies. Clin Lymphoma Myeloma 7: 106-108, 2006.

This work is licensed under a Creative Commons Attribution-NonCommercial-NoDerivatives 4.0 International (CC BY-NC-ND 4.0) License. 